Revue des patrimoines

17 | 2011

Les patrimoines de l'enseignement supérieur

\title{
La protection Monument historique : connaissance et reconnaissance des collections de l'Université de Montpellier
}

\section{Hélène Palouzié}

\section{OpenEdition}

Journals

Édition électronique

URL : http://journals.openedition.org/insitu/940

DOI : 10.4000/insitu.940

ISSN : 1630-7305

Éditeur

Ministère de la culture

Référence électronique

Hélène Palouzié, «La protection Monument historique : connaissance et reconnaissance des collections de l'Université de Montpellier », In Situ [En ligne], 17 | 2011, mis en ligne le 01 décembre 2011, consulté le 30 avril 2019. URL : http://journals.openedition.org/insitu/940 ; DOI : 10.4000/ insitu.940

Ce document a été généré automatiquement le 30 avril 2019.

\section{(c) $(1) \Theta \theta$}

In Situ Revues des patrimoines est mis à disposition selon les termes de la licence Creative Commons Attribution - Pas d'Utilisation Commerciale - Pas de Modification 4.0 International. 


\title{
La protection Monument
} historique : connaissance et reconnaissance des collections de l'Université de Montpellier

\author{
Hélène Palouzié
}

De ses grands anciens, la faculté [de médecine] n'a pas seulement recueilli un long héritage de gloire. Au cours des siècles, elle a peu a peu accumulé des richesses dont la valeur artistique le dispute souvent à l'ancienneté. Elle peut, sur bien des points, rivaliser avec plus d'un musée. Paul Delmas

$1,1921$. 


\section{Figure 1}

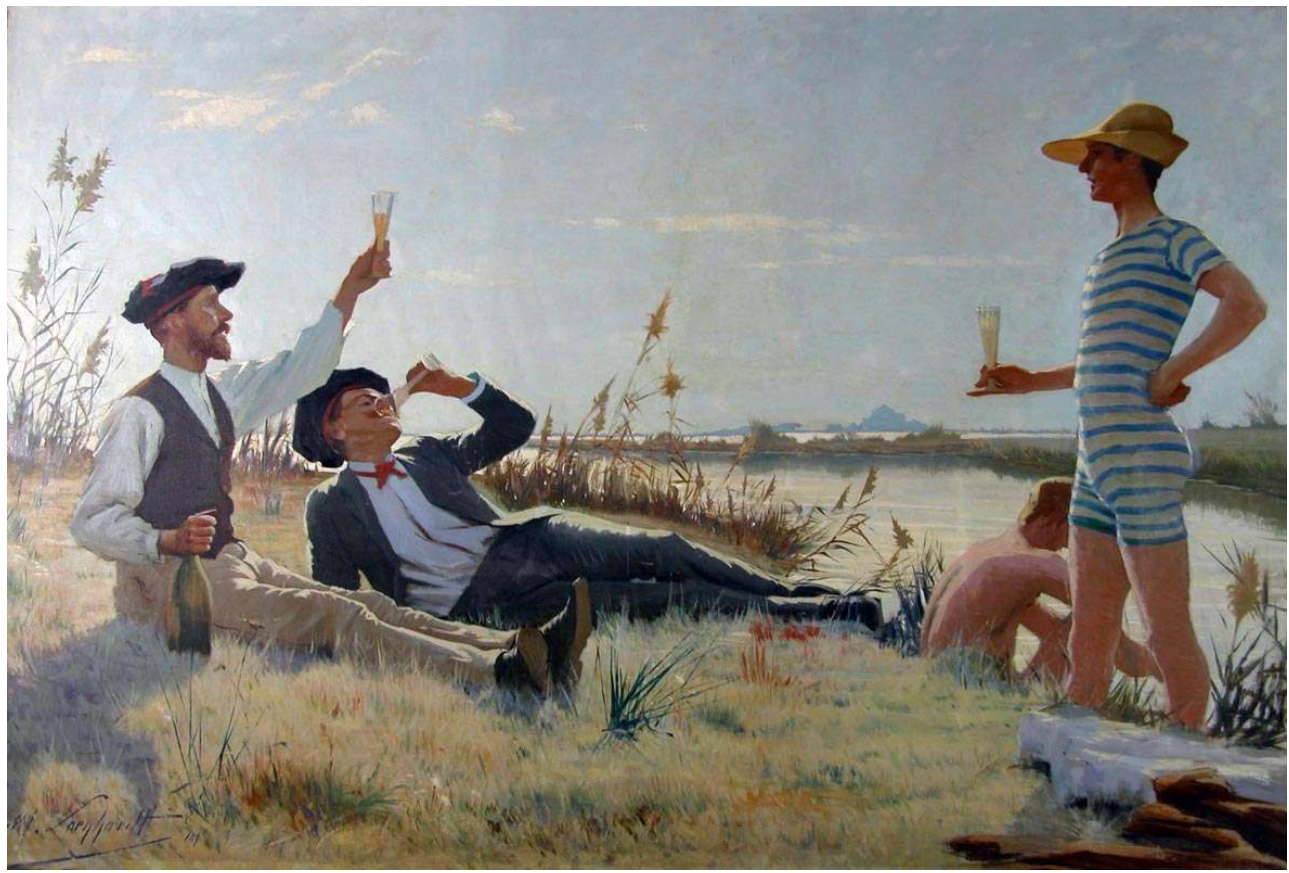

Étudiants fêtant le Vle centenaire près de la cathédrale de Maguelone (H/T, 200 x 293), Max Leenhardt (1853-1941), 1891.

(c) Toshiro Matsunaga.

\section{Figure 2}

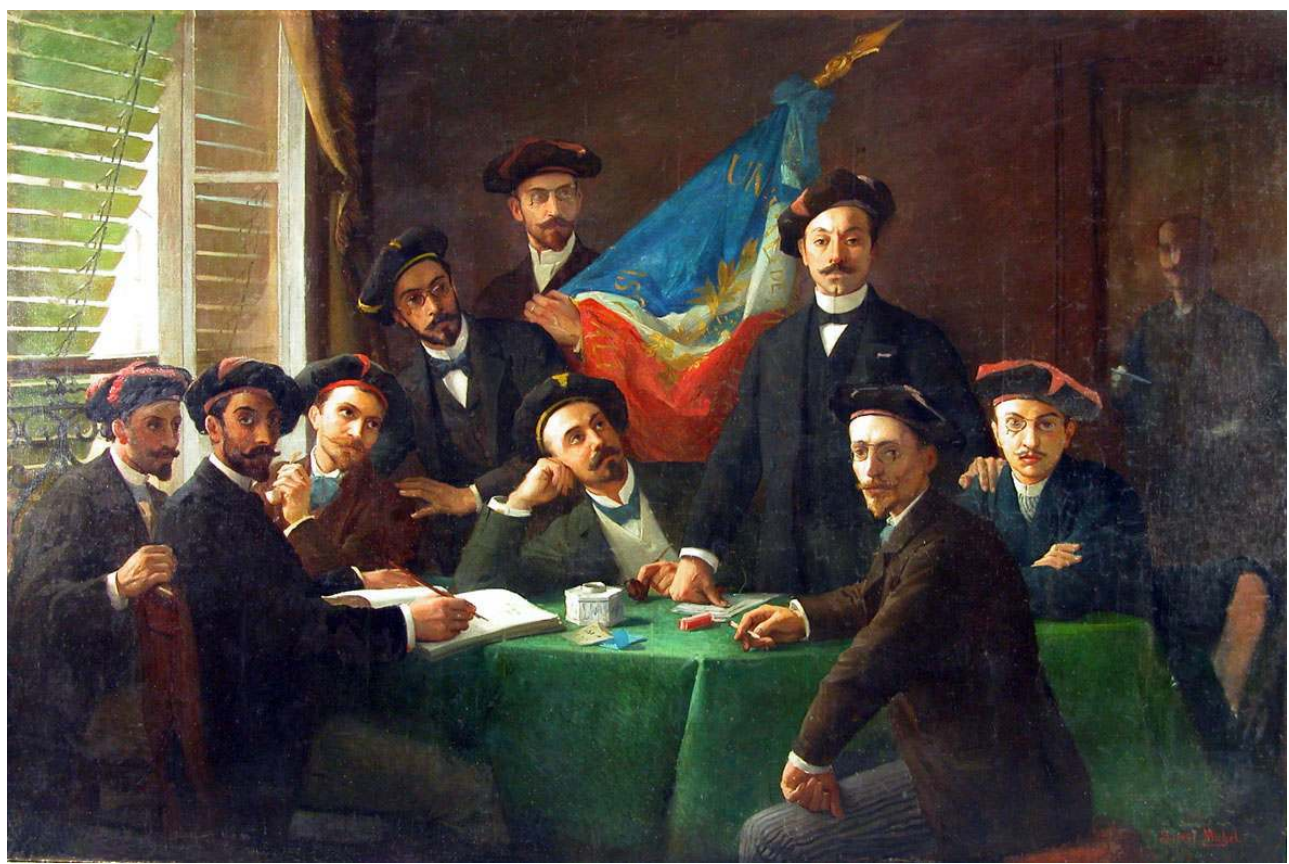

Le bureau de l'association des étudiants de Montpellier aux fêtes du Vlème centenaire (H/T, 200 x 298), Ernest Michel (1833-1902), 1892.

Toshiro Matsunaga. 
1 Lorsque le projet de Musée UM (musée de l'Université de Montpellier qui voulait rassembler en un seul lieu les trésors des trois universités) fut dévoilé à la presse en 2002, un journaliste écrivait : «Il faut reconnaître que le patrimoine des universités de la ville n'a d'égal que la misère dans laquelle il croupit ». Un patrimoine exceptionnel et menacé. (fig. $\left.n^{\circ} 1\right)$ (fig. $\left.n^{\circ} 2\right)$

2 Ville universitaire par excellence, Montpellier inaugure le premier amphithéâtre d'anatomie en 1556 avec Rondelet, le Jardin royal des plantes médicinales en 1593 grâce à Richer de Belleval, la Société royale des sciences en 1706 sous l'impulsion de Dominique Cassini. Après la Révolution Jean-Antoine Chaptal installe l'École de Santé dans l'ancien palais épiscopal, le conservatoire d'anatomie est créé, l'Hôtel-Dieu Saint-Éloi devient le Palais universitaire intégrant les collections de physique, de paléontologie et zoologie, le musée de géologie et de minéralogie, la galerie de moulages d'après l'antique et les collections de la société archéologique. Reflet du dynamisme et de l'évolution de son université, Montpellier conserve un patrimoine particulièrement riche auquel pourtant aucune étude globale n'avait encore été consacrée.

3 Le classement des monuments s'est échelonné sur plus d'un demi-siècle, en fonction des urgences de sauvegarde: collège royal de chirurgie Saint-Côme en 1945, hôpital et chapelle Saint-Charles en trois campagnes, 1947, 1977 et 1997, Jardin des plantes en 1992, faculté de médecine (ancien évêché) en 2004. Il aurait pu s'étendre à l'hôpital Saint-Éloi devenu facultés des Sciences, Lettres et Droit (actuel Rectorat) et à l'ancien collège royal de médecine, qui fut ensuite le siège de la faculté de pharmacie. Il devrait être prolongé par la protection de l'institut de botanique et de la station de biologie marine de Sète. La faculté des Lettres a reçu en 2010 le prestigieux label patrimoine du $\mathrm{XX}^{\mathrm{e}}$ siècle ${ }^{2}$. Cependant, à l'inverse, la prise de conscience de l'intérêt historique des collections qui y sont rassemblées est très récente: hormis quelques exceptions, la protection ne concernait, jusqu'à une date récente, qu'une part infime d'entre elles.

4 Corollaire $\mathrm{du}$ classement de l'immeuble, cette protection est pourtant souvent indispensable à une conservation in situ. Pour ne citer qu'un exemple, la protection des tableaux de la chapelle de l'ancien hôpital Saint-Charles a permis d'éviter leur dispersion lors de la vente du bâtiment. Ces ensembles, monument et objets, bien plus que les objets prélevés et sortis de leur contexte, ont une valeur de témoignage irremplaçable. Toutefois la reconnaissance des collections n'est venue que petit à petit, et encore ne s'agit-il que d'une reconnaissance intellectuelle, que d'un souhait de conservateur, la loi obligeant à distinguer, pour les protéger, chaque objet un à un, ignorant toute valeur d'ensemble ou issue du contexte. Leur classement est en fait une question de culture et de volonté institutionnelle. La législation sur les monuments historiques peut apparaitre compliquée ou contraignante, mais tout le monde l'utilise; quand on affiche le label monument historique, par l'identification culturelle sur le plan national qu'il sous entend, les comportements aussi changent ${ }^{3}$.

5 Force est de constater que jusqu'en 2004, peu de collections universitaires étaient classées au titre des Monuments Historiques. Le premier classement date de 1913, année de la loi fondatrice des Monuments historiques, et concerne 6000 dessins et estampes de la collection Atger ainsi que les manuscrits de la bibliothèque de la faculté de médecine de Montpellier. Ce n'est que bien plus tard que furent classés d'autres grands ensembles, comme les collections de tableaux et dessins de la faculté de lettres de Lyon en 1973, celles de l'Institut de géologie de Rennes en 1990 ou encore les collections anatomiques 
Delmas - Orfila-Rouvière de Paris V en 1992. Partant du postulat qu'un objet Monument Historique est indissociable de son contexte, il est intéressant, au regard des collections des universités de Montpellier, de souligner l'évolution qu'a connue la protection, de la conservation de l'objet exceptionnel, jusqu'à la conservation d'une collection et à la sauvegarde d'un lieu de mémoire. Cette démarche de classement des collections universitaires est novatrice et unique en France à une échelle aussi importante ${ }^{4}$. (fig. $\mathbf{n}^{\circ} 3$ ) (fig. $\left.n^{\circ} 4\right)$

Figure 3

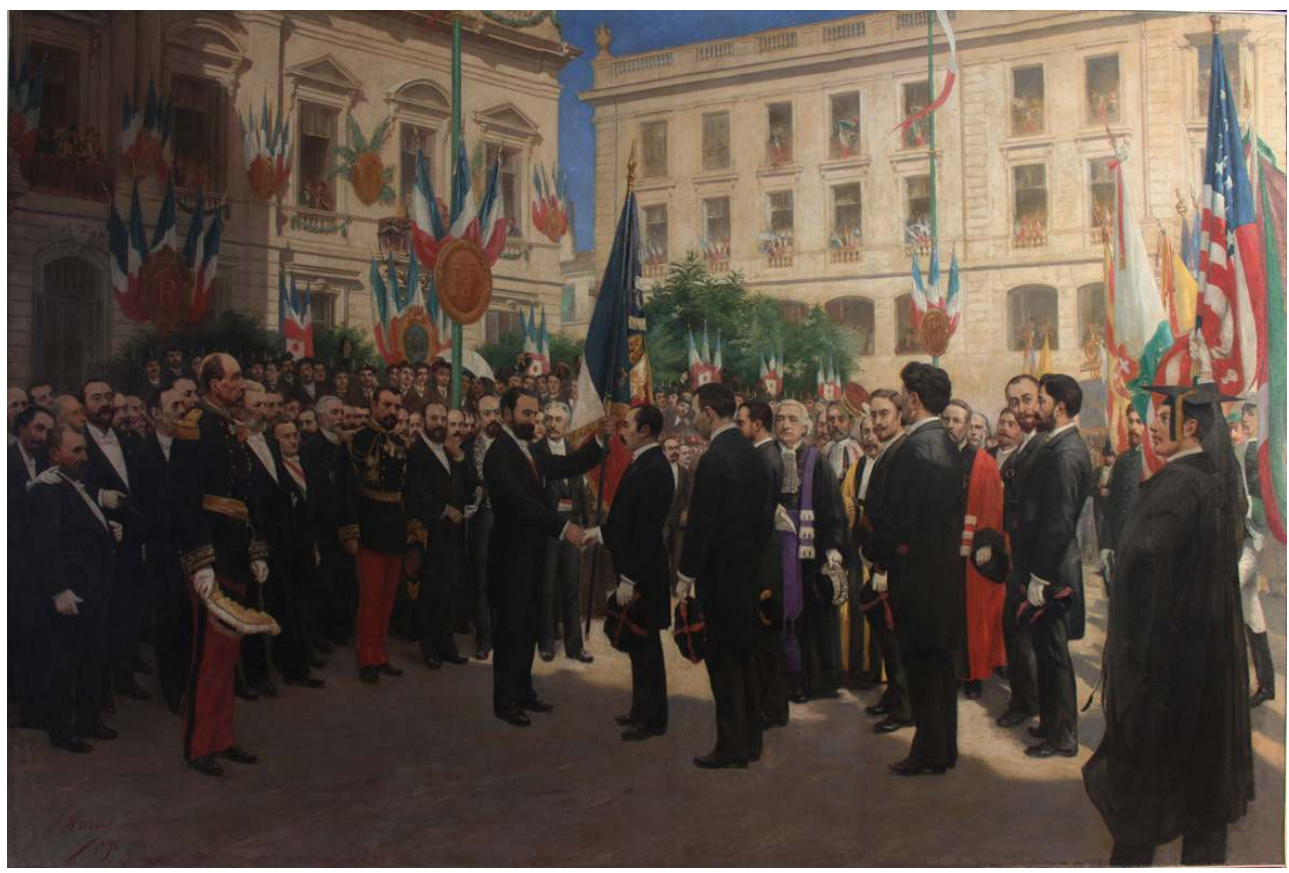

La remise du drapeau à I'Association générale des étudiants par le président de la République Sadi Carnot (H/T, 200 x 300), Édouard Marsal (1845-1929), 1891.

(c) Toshiro Matsunaga. 


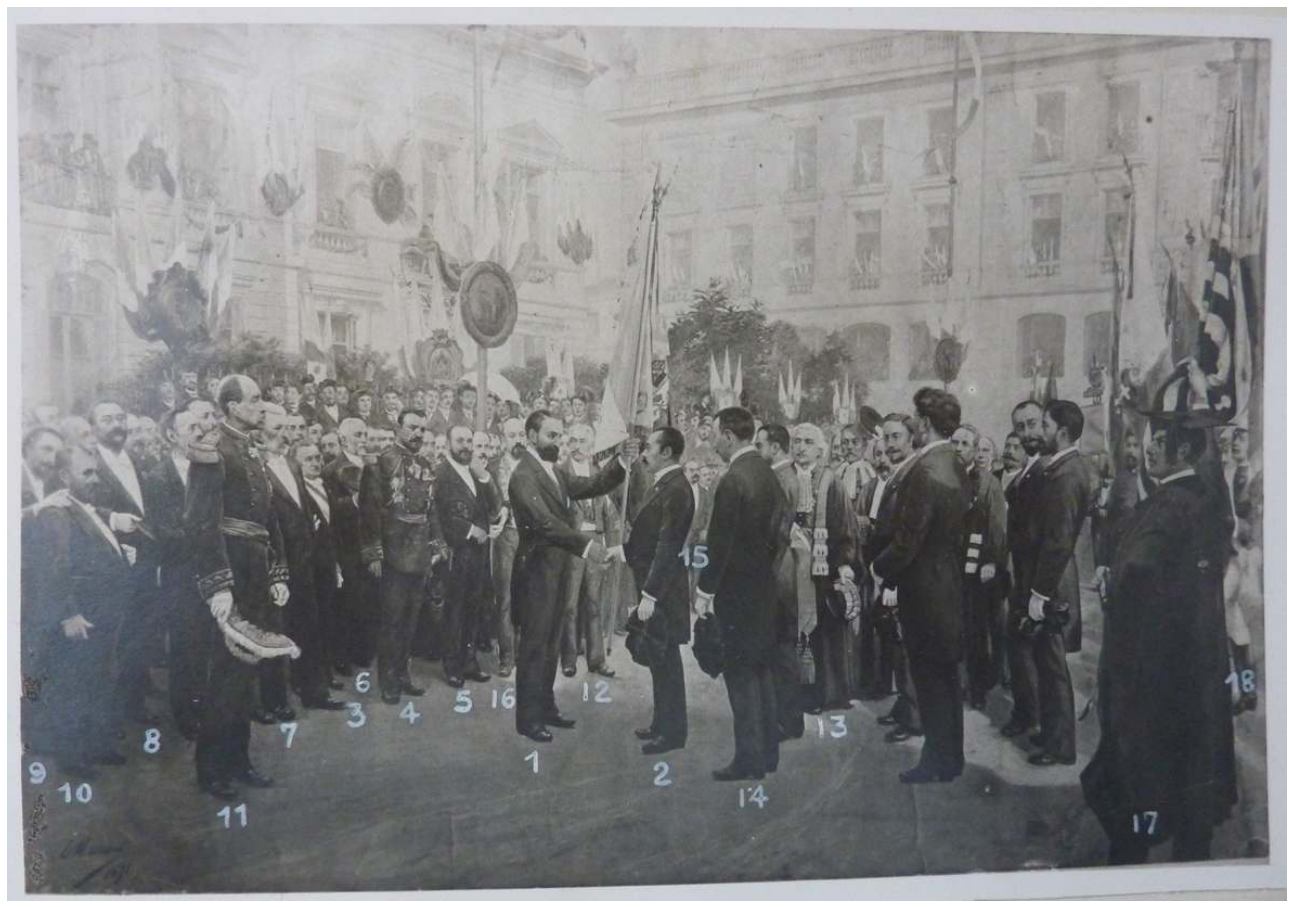

Extrait de : ROUZAUD, Henri. Montpellier 1890. Les fêtes du Vle centenaire de l'Université. Montpellier : Camille Coulet, 1891.

6 L'inventaire des collections, très partiel avant les études en vue d'une protection au titre des Monuments historiques, ne cesse de s'étendre, car cette accumulation d'objets de toute nature recèle un pouvoir d'attraction qui s'amplifie lorsque l'on en décèle les multiples sens. L'évolution des disciplines rend encore plus impérieuse la nécessité de répertorier ces objets de savoir qui ne sont plus seulement l'apanage d'une institution, mais s'inscrivent désormais dans le patrimoine historique, la mémoire collective. Outils de connaissance et œuvres d'art se distinguent et se fondent en un même ensemble pour la plus grande gloire de l'institution. Suivant la distinction établie par Jean-Antoine Chaptal ${ }^{5}$, entre collection à visées didactiques et collection "mémorial », les collections universitaires concernent aussi bien des collections vivantes, c'est-à-dire utilisées pour l'enseignement, mais aussi de façon plus spécifique, des collections "mémorial», collections dont l'usage a cessé mais qui participent également à la transmission du savoir et qui sont liées à l'identité, au prestige de l'institution. Ce sont ces collections qui s'inscrivent dans le "génie du lieu» pour lesquelles il faut trouver une solution patrimoniale. Le seul historien a s'être soucié de sa conservation est certainement le professeur Louis Dulieu ${ }^{6}$, en évitant notamment la disparition des galeries de portraits des hôpitaux et en soulignant l'intérêt des collections de portraits des professeurs de chaque faculté.

7 C'est dans une perspective historique, centrée sur l'origine et la pérennité de ce rassemblement, que peut être appréhendé le classement des collections universitaires et le plan de sauvegarde mis en œuvre ces cinq dernières années. 


\section{La redécouverte des collections : l'année 1890 et le $\mathrm{Vl}^{\mathrm{e}}$ centenaire de l'Université de Montpellier}

Figure 5

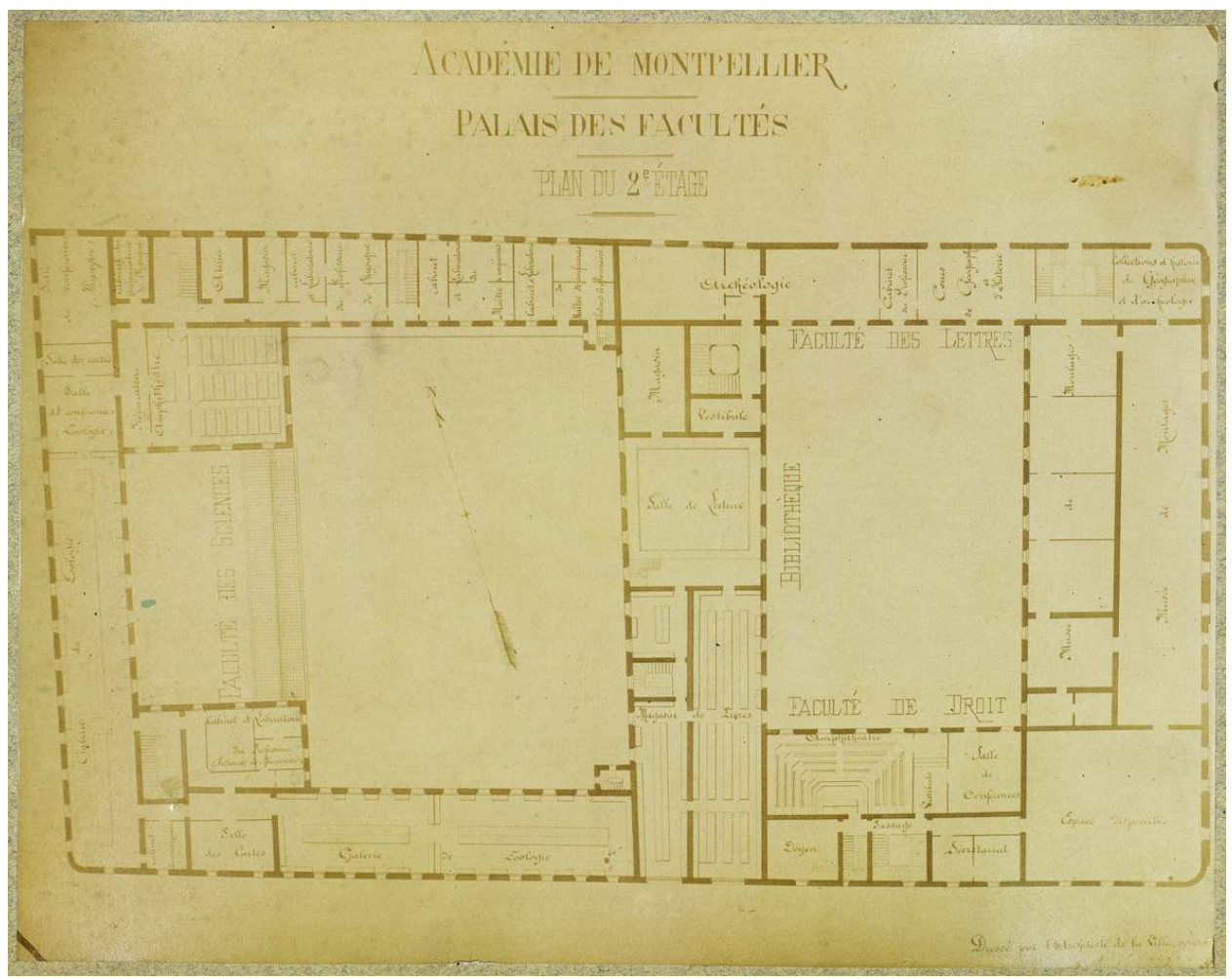

Palais des Facultés des Sciences, Lettres et Droit, plan dressé par l'architecte de la ville Anthony Kruger; détails des salles de présentation des moulages, des collections de géologie, musée de physique. Atlas des batimens de l'Hôtel dieu Saint Eloi à Montpellier, AD 34 - 1H dépôt 2013.

(c) Archives départementales de l'Hérault.

La redécouverte quelque peu fortuite, hasard de la recherche, de six tableaux créés pour la célébration et la commémoration du VI ${ }^{\mathrm{e}}$ centenaire de l'université est révélatrice de la nécessité de replacer les collections dans leur contexte historique pour mieux en cerner l'intérêt et assurer leur conservation. Significatif du mécanisme de redécouverte, cet exemple, que l'on pourrait multiplier grâce aux études menées depuis plusieurs années par la DRAC, (conservation régionale des monuments historiques), en partenariat avec l'université et les responsables des collections, a valeur de symbole ${ }^{7}$ : l'oubli, la méconnaissance sont bien souvent synonymes de dégradation, de déplacement, voire de destruction. (fig. $\mathbf{n}^{\circ} \mathbf{5}$ )

L'Université de Montpellier, créée le 26 octobre 1289 par la bulle du pape Nicolas IV, célèbre avec faste son $\mathrm{VI}^{\mathrm{e}}$ centenaire en 1890, du 21 au 26 mai, en présence du président de la République Sadi Carnot, du ministre de l'instruction publique, Léon Bourgeois, du directeur de l'enseignement supérieur, Louis Liard, et de Gaston Boissier, de l'Académie française. Une toile emblématique vient d'être redécouverte, toujours conservée in situ, dans l'ancien palais universitaire, actuel rectorat. Dans la lignée de l'Apothéose d'Homère (Ingres, 1827) ou de l'Hémicycle de l'École des Beaux-Arts (Delaroche, 1837), l'université 
commande pour la salle des fêtes de son palais l'allégorie des Savoirs du monde et de l'université de Montpellier ; l'artiste choisi est le peintre montpelliérain Ernest Michel, élève de Cabanel, connu pour ses décors du théâtre et du palais de Justice de Montpellier. Ce tableau symbolise l'union des Arts et Sciences, reflétant le projet d'une université républicaine sur le modèle de l'université pluridisciplinaire de Berlin, l'université des savoirs, regroupée sous un même toit et sous une même autorité, mettant un terme provisoire à la fragmentation du système des facultés napoléoniennes et renouant avec l'organisation de l'université médiévale. Candidate au statut de ville universitaire du sud de la France, Montpellier souhaite alors promouvoir l'université régionale par l'édification d'un palais universitaire (la faculté de médecine et l'école de pharmacie - qui sera faculté en 1920 - échappent à ce regroupement). Toutes les institutions se sont associées, préfecture, conseil général, mairie, sociétés savantes pour faire de cette fête intellectuelle un événement grandiose et populaire, imaginé par Alexandre Germain (1809-1887), doyen de la faculté des Lettres et membre de l'académie des sciences et des lettres de Montpellier. L'objectif de ces fêtes du VI ${ }^{\mathrm{e}}$ centenaire, célébrant la renaissance de l'université, est de mettre en scène la dimension universitaire de la ville, de se distinguer auprès des élites nationales et d'emporter la décision du gouvernement. $\mathrm{Ce}$ tableau fait écho à l'organisation que l'université souhaite mettre en place avec ses cinq facultés, médecine, droit, lettres, sciences et pharmacie, en inaugurant le palais de l'université et le musée des moulages le samedi 24 mai 1890 dans l'ancien hôpital SaintÉloi, réaménagé par l'architecte de la ville Anthony Kruger. Plan Campus avant la lettre le parallèle avec le projet actuel de fusion des trois universités est saisissant et riche d'enseignements. (fig. $\left.\mathbf{n}^{\circ} \mathbf{6}\right)\left(\right.$ fig. $n^{\circ} 7$ )

Figure 6

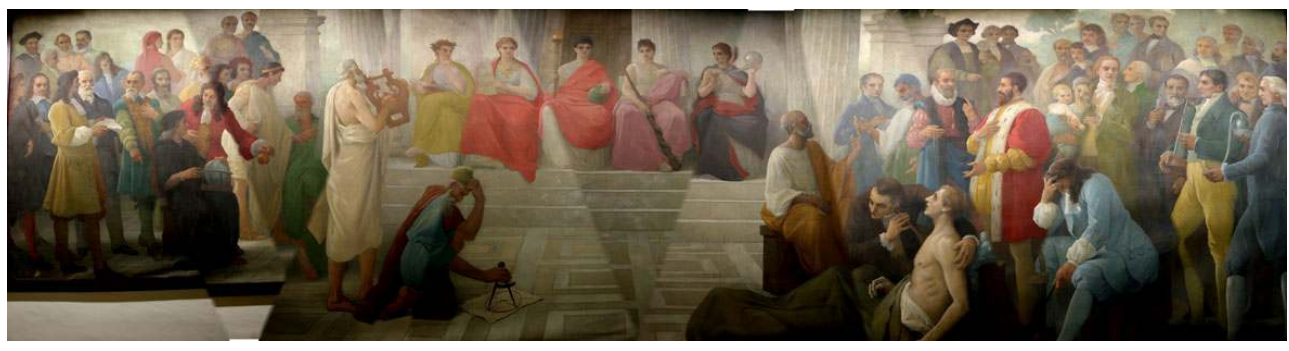

Les savoirs du monde et de l'université de Montpellier (H/T, 300 x 900), Ernest Michel (1833-1902), 1890. (c) Service des Collections, Montpellier II. 


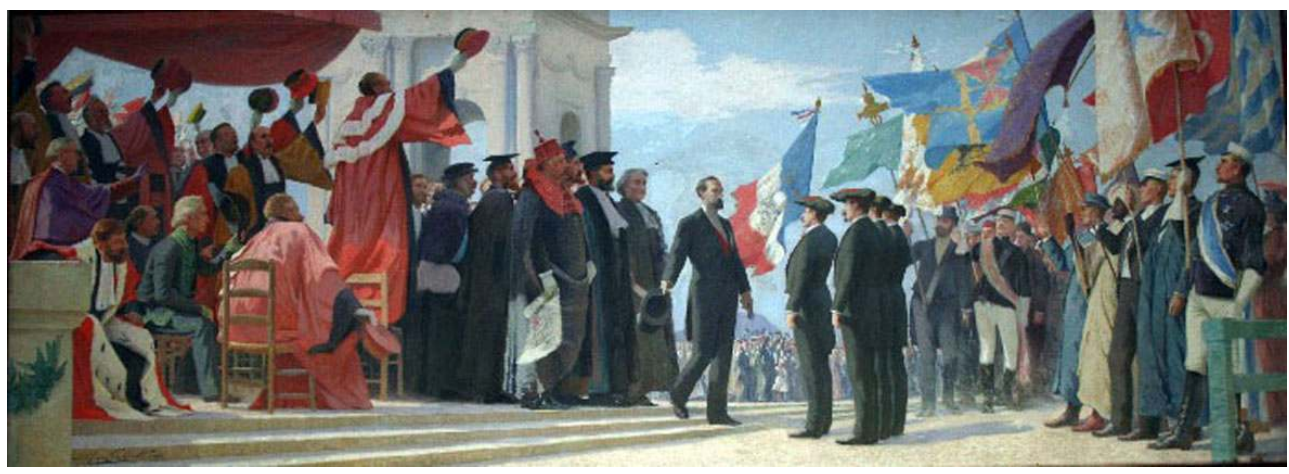

Le salut des drapeaux sur la place du Peyrou (H/T, 300 x 700), Max Leenhardt (1853-1941), 1892.

(c) Service des Collections, Montpellier II.

10 Ce tableau fait partie des toiles commandées à trois artistes montpelliérains renommés au $\mathrm{XIX}^{\mathrm{e}}$ siècle et aujourd'hui injustement oubliés, Ernest Michel ${ }^{9}$ (1833-1902), Max Leenhardt ${ }^{10}$ (1853-1941) et Édouard Marsal ${ }^{11}$ (1845-1929). Contemporains d'autres artistes montpelliérains tels que Frédéric Bazille (1841-1870), Charles Matet (1791-1870), Auguste Glaize (1807-1893), Jean-Pierre Montseret (1813-1888) et bien sûr d'Alexandre Cabanel (1824-1889), dont ils furent les élèves, ils réalisèrent aussi de nombreux portraits pour la galerie des professeurs de l'université.

11 La redécouverte de trois toiles que l'on croyait disparues, mises au rebut et roulées dans un réduit de la faculté de médecine, a permis de mettre en relation les œuvres commémorant le centenaire. La première, d'Édouard Marsal, La remise du drapeau à l'Association générale des étudiants par le président de la République Sadi Carnot sur la place de la Préfecture le vendredi 23 mai 1890, ornait l'escalier d'honneur de la faculté de médecine ${ }^{12}$. À cette première cérémonie assistent Chancel ${ }^{13}$ recteur de l'Académie, les doyens Vigié (faculté de droit), de Rouville (faculté des sciences), Castets (faculté de lettres), Castan (faculté de médecine) et le directeur de l'école de pharmacie (Diacon). On peut reconnaître, en outre, le préfet Pointu-Nores, Eugène Lisbonne, président du conseil général, Alexandre Laissac, maire de Montpellier. Elle complète l'ensemble formé par les deux toiles immortalisant la célébration de l'après-midi, Le salut des drapeaux sur la place du Peyrou le 23 mai : l'une d'Ernest Michel pour le Conseil général est conservée aux archives départementales, l'autre de Max Leenhardt, destinée à la salle des Fêtes du palais universitaire est au Rectorat. Le cérémonial prévoyait que la bannière de l'association des étudiants serait entourée de toutes les bannières des délégations des universités étrangères et que les étudiants défileraient ensuite devant le président de la République, reconnaissables à leur faluche, ornée de liserés de différentes couleurs selon les disciplines : rouge pour le droit, verte pour la pharmacie, grenat pour la médecine, jaune pour les lettres. À côté de ces tableaux officiels, et commandés aux mêmes peintres, les deux autres tableaux retrouvés, Le Bureau de l'association des étudiants de Montpellier aux fêtes du VI centenaire (Ernest Michel) et Étudiants fêtant le VI centenaire près de la cathédrale de Maguelone (Max Leenhardt), rappellent la fête des étudiants du 26 mai 1890. Ce sont des donations des artistes pour le Cercle des étudiants (actuel Pavillon populaire) qui venait d'être construit par l'architecte Carlier pour l'association des étudiants nouvellement créée, l'A.G.E.M. et présidée par Jean-François Guy. 
Figure 8

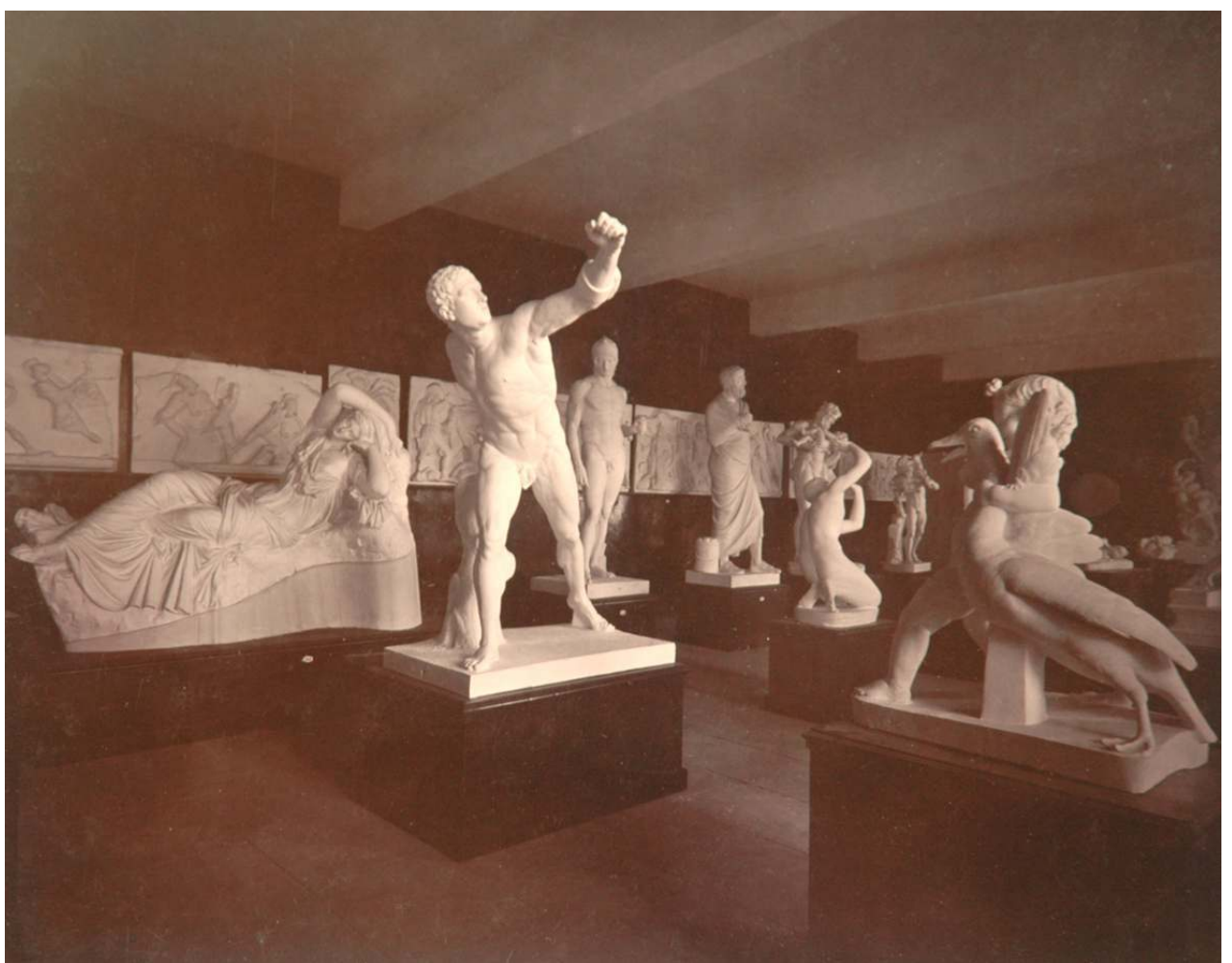

Photo de la collection de moulages lors de son installation en 1890 dans le palais de l'Université, ancien hôpital Saint-Éloi. Atlas des batimens de l'Hôtel dieu Saint Eloi à Montpellier, AD 34 - 1H dépôt 20 13.

(c) Archives départementales de l'Hérault. 


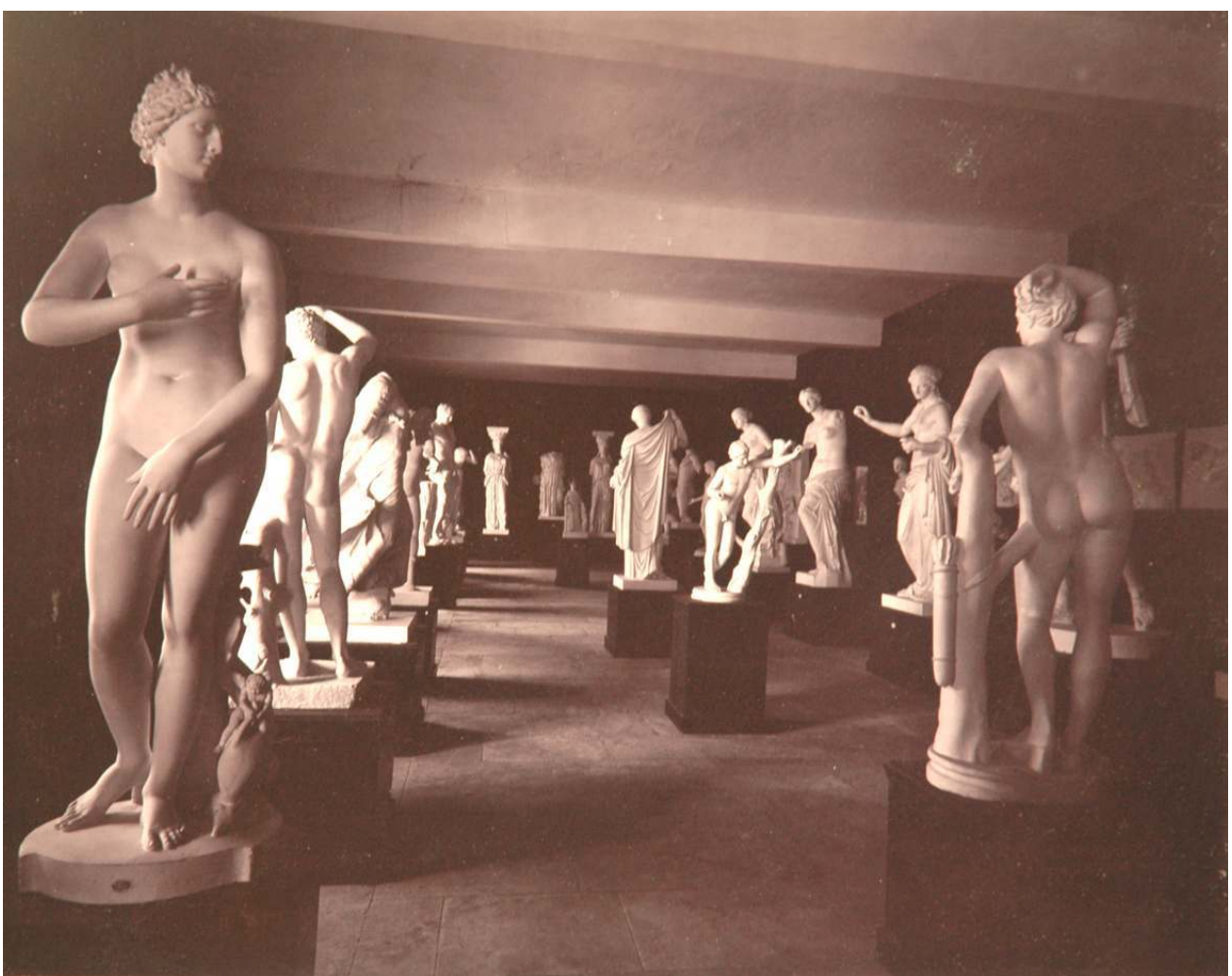

Photo de la collection de moulages lors de son installation en 1890 dans le palais de l'Université, ancien hôpital Saint-Éloi. Atlas des batimens de l'Hôtel dieu Saint Eloi à Montpellier, AD 34 - 1H dépôt 20 13.

(c) Archives départementales de l'Hérault.

L'année 1890 est capitale car elle est celle de l'aboutissement de projets d'importance pour la nouvelle université : la création par Ferdinand Castets ${ }^{14}$ (1838-1911) du Musée des moulages et celle de l'Institut de botanique par Charles-Marie Flahault (1852-1935). (fig. $n$ •8) (fig. n'9) $^{\circ}$

Comme le suggérait le ministre de l'Instruction publique et des Beaux-Arts en 1889, la galerie de moulages, installée dans le nouveau palais des facultés, fut inaugurée lors des fêtes du sixième centenaire par le président de la République ${ }^{15}:$ « Depuis longtemps mon administration a l'intention de créer à Montpellier une galerie d'archéologie classique (moulages d'après l'antique); une telle collection est la parure d'une Faculté des Lettres ; une somme importante est tenue en réserve pour la Faculté de Montpellier. Mon vif désir est que cette galerie soit constituée complètement pour les Fêtes du Centenaire ${ }^{16} »$. Le catalogue du musée de moulages, rédigé par Ferdinand Castets, est édité quelques jours avant les fêtes $d u \mathrm{VI}^{\mathrm{e}}$ centenaire. Des documents nous renseignent sur la présentation d'origine du musée destiné à être ouvert au public: "les salles sont peintes en rouge sombre et ornées de frises à l'antique, les socles des statues sont brun Véronèse ${ }^{17}$ ». 


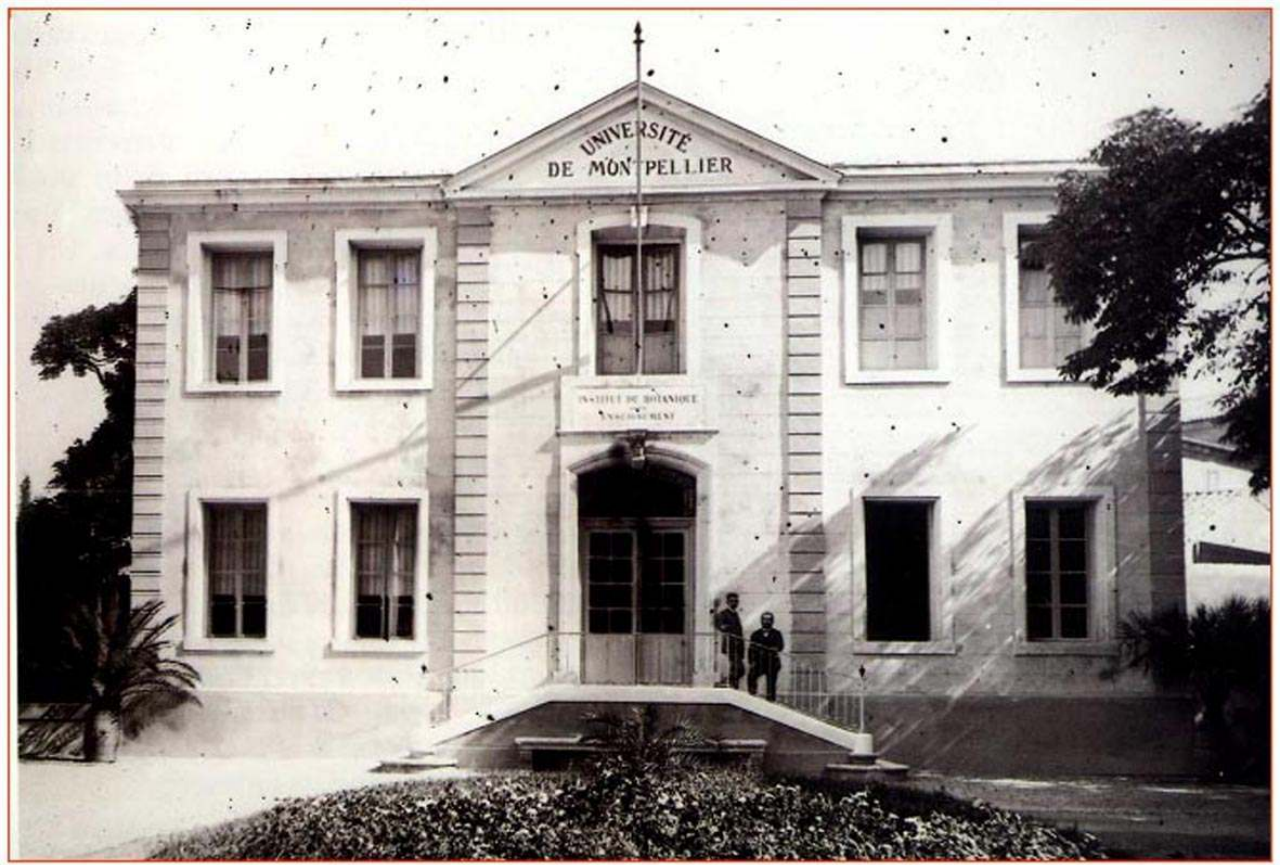

Institut de botanique de Charles Flahault, 1890

(c) DRAC Languedoc-Roussillon.

Figure 11

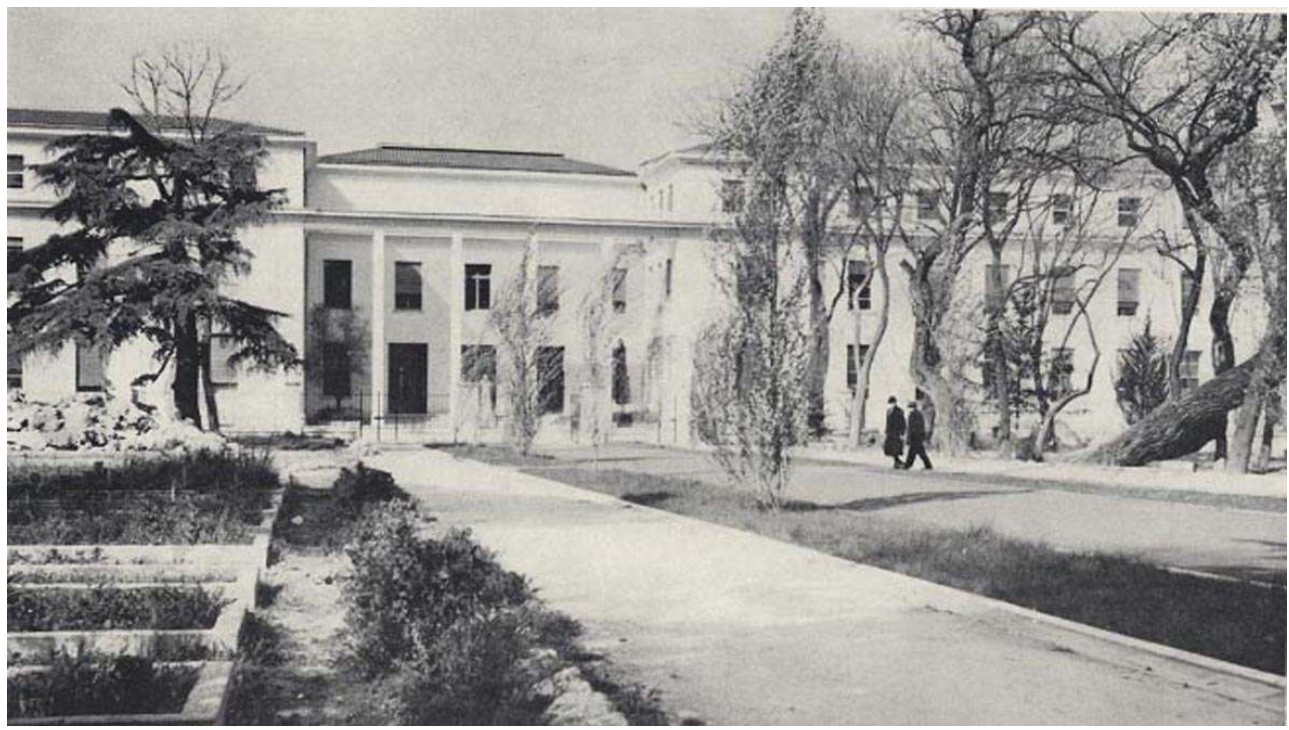

Institut de botanique actuel, Jean de Richemond, 1959.

(c) DRAC Languedoc-Roussillon. 
Figure 12

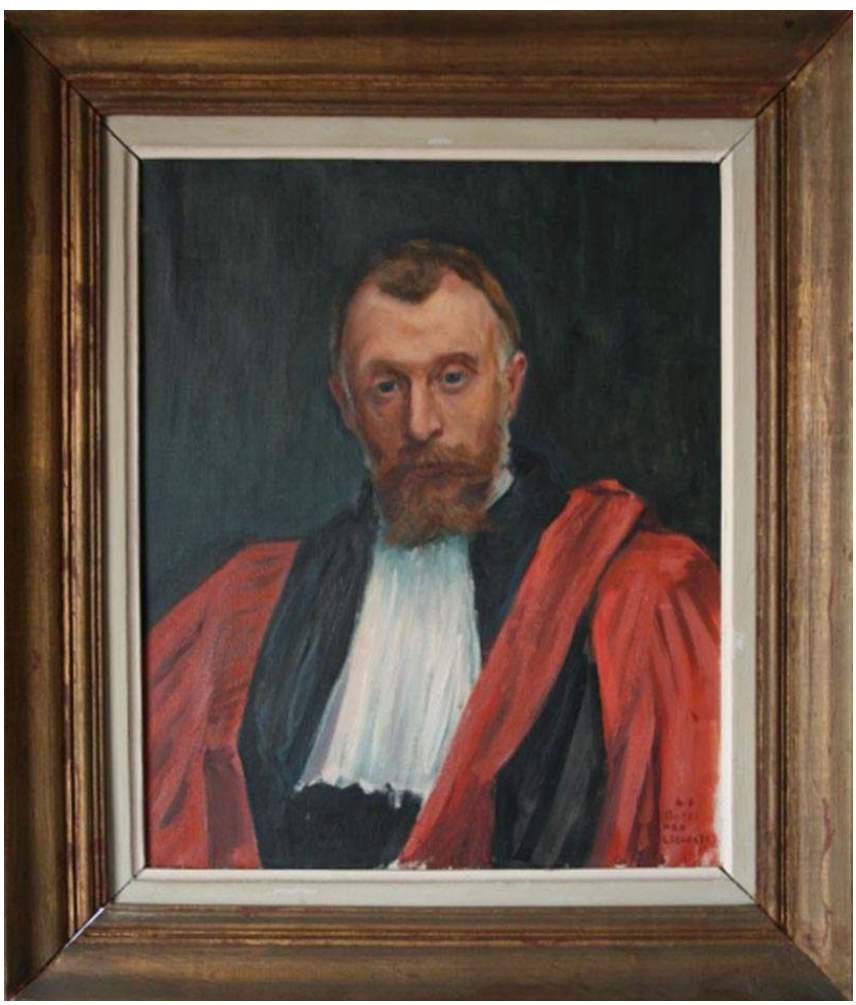

Portrait de Charles Flahault, Georges Dezeuze (1905-2004) d'après Leenhardt, 1936 (c) Service des Collections, Montpellier II. 
Figure 13

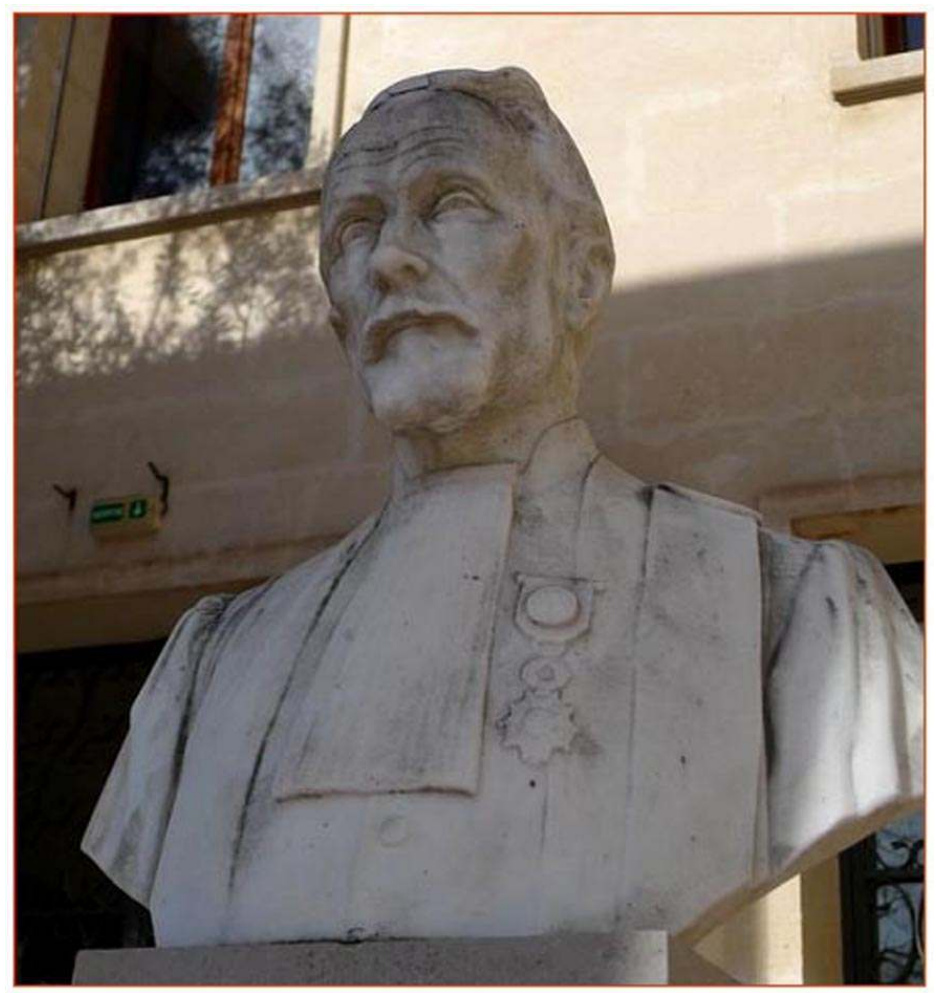

Monument à Charles Flahault, Jean Magrou, 1936.

(c) Service des Collections, Montpellier II.

Par la création de l'Institut de Botanique, Charles-Marie Flahault (1852-1935) réunit les trois instituts de recherche sur la botanique des facultés de médecine, pharmacie et sciences et les installe en 1890 dans trois maisons du XVIII ${ }^{e}$ siècle récemment achetées et mitoyennes au nord du Jardin des plantes. L'Institut aménagé par l'architecte Devic regroupe locaux scientifiques, laboratoires, ateliers, mais aussi l'une des plus grandes collections d'herbiers. Le vestibule d'honneur est orné des toiles peintes et offertes par Leenhardt, toujours conservées: Herborisation d'étudiants dans la garrigue et Laboratoire de l'ancien institut de botanique. Il était prévu un musée d'étude pour le public, le musée Rondelet. Le monument à Charles Flahault, réalisé par le sculpteur biterrois Jean Magrou et inauguré le 11 juillet 1936, orne l'actuel parvis de l'Institut sur le Jardin des plantes ${ }^{18}$. Son gendre, Louis Emberger (1897-1969), fonde plus tard, en 1959, le second et actuel Institut de botanique, réalisé par l'architecte de l'université : Jean de Richemond. (fig. $\mathbf{n}^{\circ}$ 10) (fig. $\left.n^{\circ} 11\right)$ (fig. $\left.n^{\circ} 12\right)$ (fig. $\left.n^{\circ} 13\right)$

15 La redécouverte des œuvres du $\mathrm{VI}^{\mathrm{e}}$ centenaire, replacées dans leur contexte historique, permet de porter un regard nouveau sur l'université, son organisation et son mode d'enrichissement. Elles participent du mouvement de reconnaissance engendré par la protection au titre des Monuments historiques, stimulant et fédérant la recherche sur le patrimoine universitaire de Montpellier. En fonction des diverses campagnes de classement des collections, des études voient le jour en collaboration avec les institutions parisiennes : mémoire de l'Institut national du patrimoine sur la statuaire du Jardin des Plantes (Julie Tugas, 2007), mémoires de l'École du Louvre sur les momies du 
conservatoire d'anatomie (Nicolas de Larquier, 2009), sur le musée des moulages (Soline Morinière, 2010).

\section{Reconnaissance des collections universitaires : la protection Monument historique}

La volonté de labelliser ces objets de savoir constitue le point de départ de reconnaissance d'un patrimoine, elle est un gage pour l'avenir, afin d'éviter, un jour, leur dispersion. La protection au titre des Monuments historiques concerne en premier lieu des œuvres en déshérence, c'est-à-dire généralement non gérées par des conservateurs spécialistes et peu accessibles au public. Toutefois, il ne suffit pas qu'un objet soit riche d'histoire pour que son classement aille de soi. L'évolution de la protection a permis de ne plus reconnaître comme seuls dignes de classement les objets exceptionnels, mais de prendre en compte des collections qui, par leurs liens à l'édifice qui les abrite, font qu'un objet sans grande valeur marchande peut devenir Monument historique. Au reste, si la notion de protection s'élargit avec la nécessité de sauvegarde, c'est avant tout une meilleure connaissance des objets qui en détermine l'intérêt. Seule, la réalisation d'études exhaustives permet de connaitre les différentes phases d'aménagement d'un lieu et autorise la réflexion sur la conservation d'un fonds. L'étude des collections des facultés de Montpellier, qui n'avait jamais été envisagée dans sa globalité, révèle le lien étroit entre des œuvres, de nature, d'origine et de qualité différentes, mais qui toutes participent à la constitution de ce panthéon universitaire: manuscrits, dessins, estampes, peintures, sculptures, aquarelles sur vélins, planches anatomiques, instruments d'astronomie, galerie d'anatomie, galerie de moulages, herbiers, droguier, collections photographiques et archives sont la mémoire tangible d'un enseignement pluridisciplinaire. Si le temps fort du rassemblement des collections se situe au tournant du siècle des Lumières, l'enrichissement des collections ne cesse de croitre tout au long du XIX ${ }^{e}$ siècle, preuve du dynamisme de l'université. L'histoire du classement de la collection d'anatomie de la faculté de médecine et de la collection de moulages de la faculté de Lettres a valeur d'exemple : il n'y a pas de solution miracle pour sauvegarder ces objets qui ont perdu leur efficacité et dont le statut patrimonial supplante la valeur d'usage, jusqu'alors gage de conservation. (fig. $\left.n^{\circ} 14\right)\left(\right.$ fig. $\left.n^{\circ} 15\right)\left(\right.$ fig. $\left.n^{\circ} 16\right)\left(\right.$ fig. $\left.n^{\circ} 17\right)$

\section{La collection d'anatomie de la faculté de médecine}


Figure 14

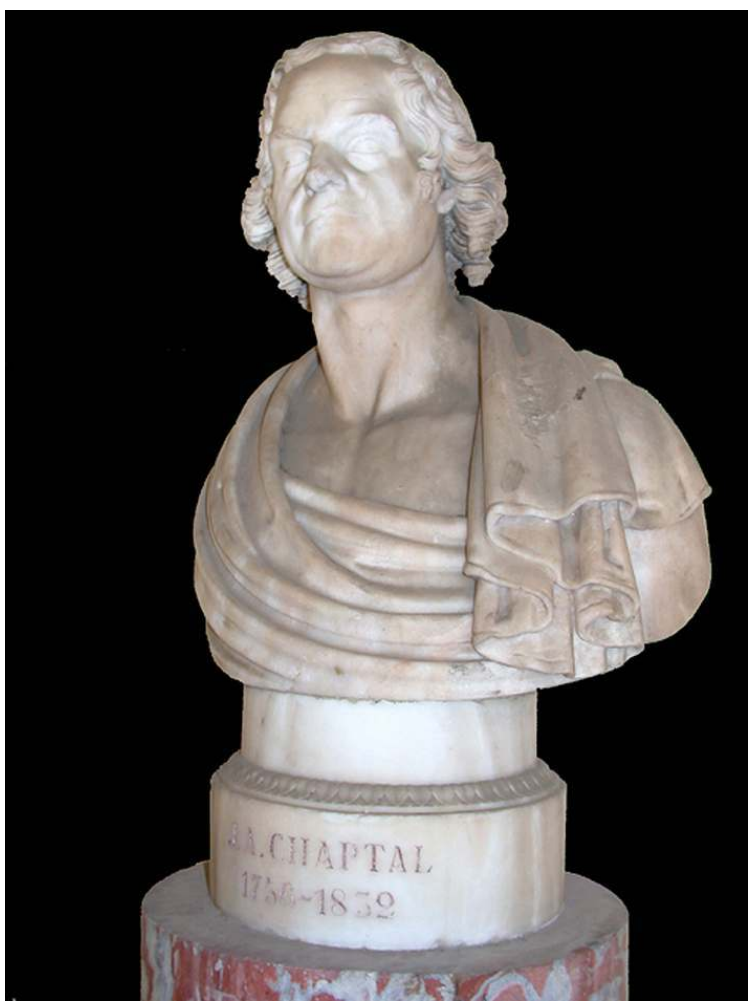

Jean-Antoine Chaptal 1756-1832, Jean-Baptiste Comolli (1775-1830), sculpteur turinois, élève de Canova, marbre, 1803.

(c) DRAC Languedoc-Roussillon. 
Figure 15

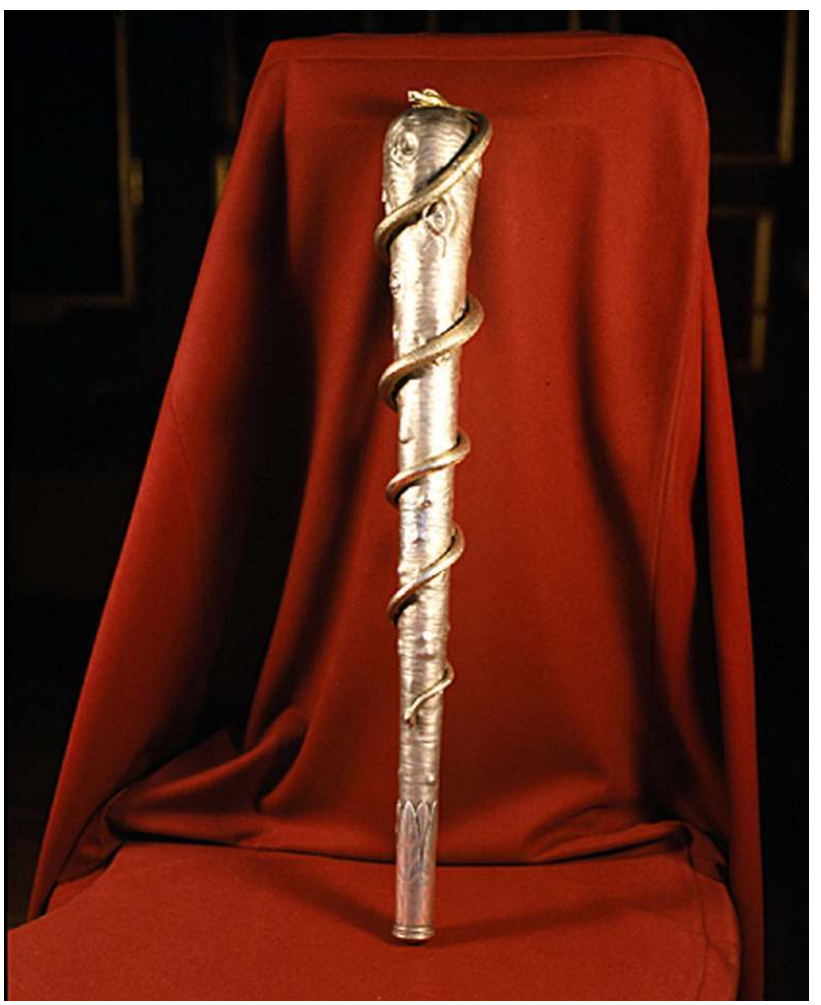

Masse d'Esculape. Jean-Baptiste Odiot, 1803.

(c) DRAC Languedoc-Roussillon. 


\section{Figure 16}

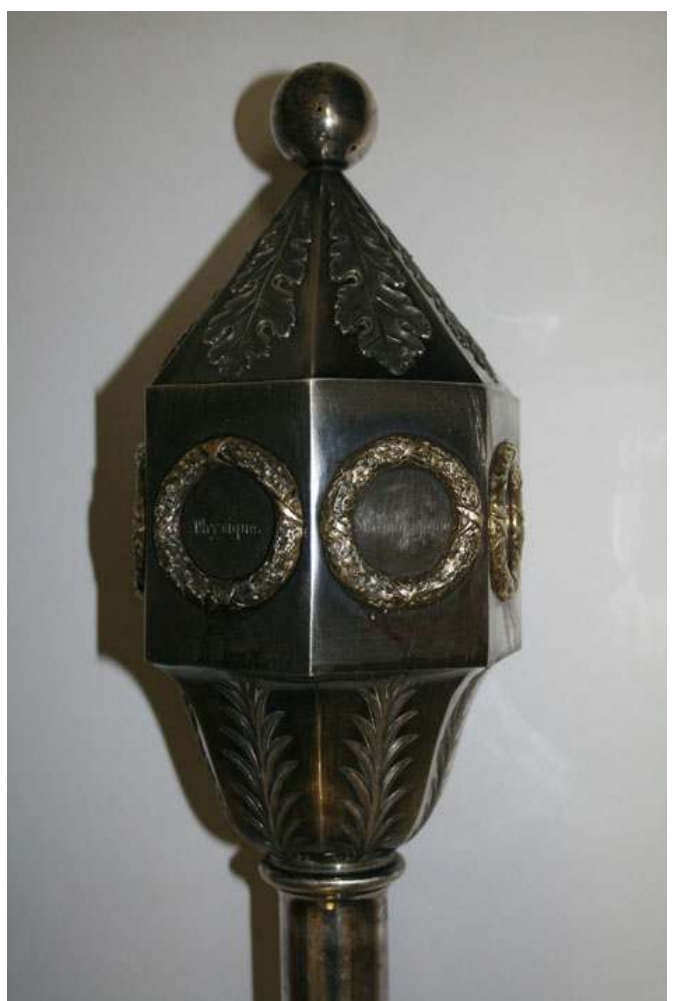

Masse de la faculté des sciences, Placide Bouet, vers 1810.

(c) Service des Collections, Montpellier II. 


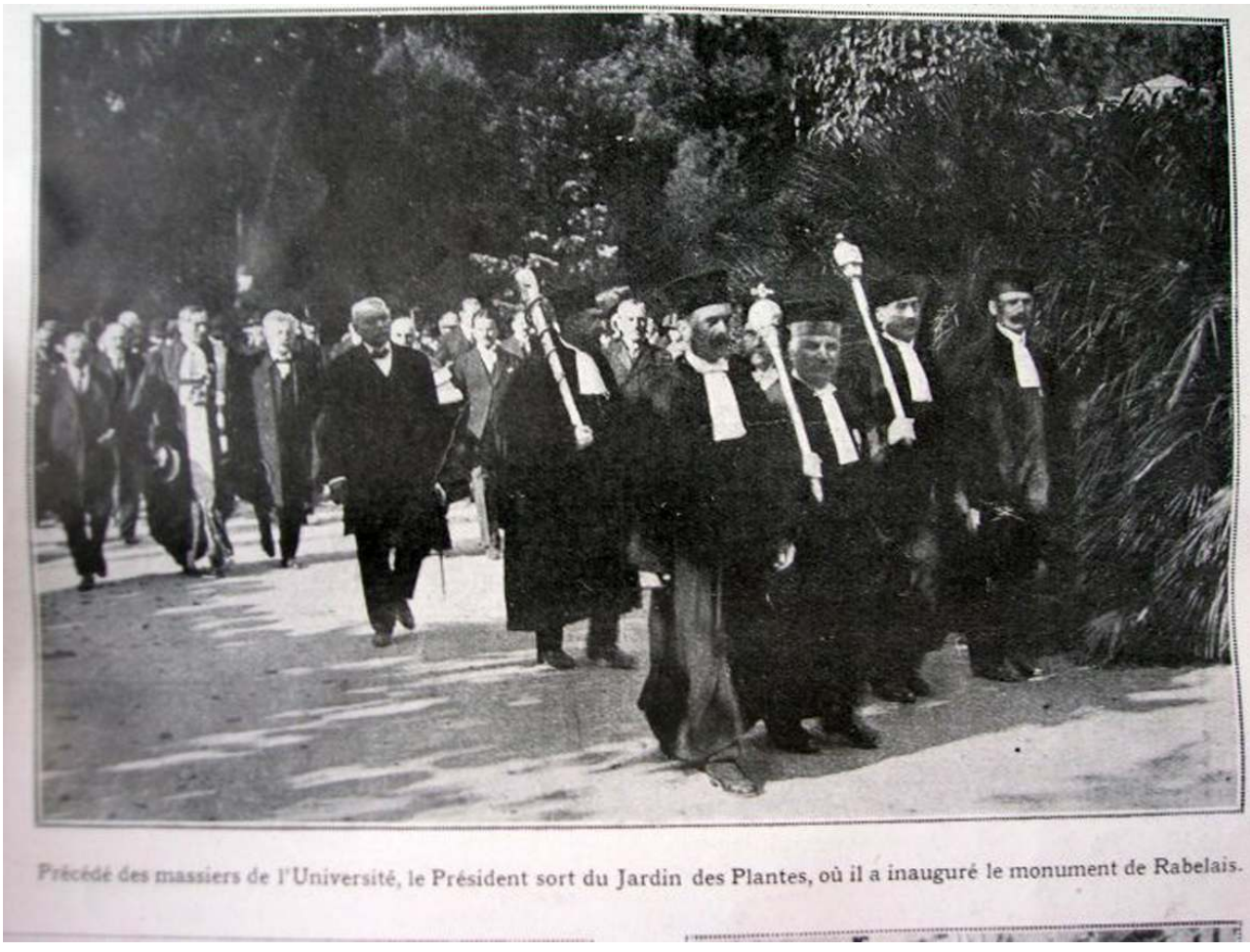

Inauguration lors des fêtes du $\mathrm{Vl}^{\mathrm{e}}$ centenaire.

(C) Julie Tugas.

17 Suite à la demande de protection du conservatoire d'anatomie, l'étude des œuvres de la faculté de médecine a permis de révéler des aspects méconnus de cette collection, de mesurer l'importance du rôle de Chaptal et de redonner tout leur lustre à des œuvres de premier plan tombées dans l'oubli. Jean-Antoine Chaptal ${ }^{19}$ (1756-1832) installe l'École de santé de Montpellier le 22 avril 1795 dans l'ancien palais épiscopal - collège Saint-Benoît qu'il considérait comme le plus beau et le plus ancien monument d'instruction que possède la France. Par ses fonctions de ministre de l'Intérieur sous Bonaparte de 1800 à 1804, il est à l'origine de l'organisation des dépôts d'objets de sciences et d'art et de la création des musées de province. Il rêva, à l'instar de Quatremère de Quincy (1755-1849), de réunir dans un même édifice les arts et les sciences, par la création d'un muséum temple de la science voué à l'enseignement, rassemblant collections d'anatomie, bibliothèque, galerie de tableaux, sculptures et dessins. Le noyau des collections a été constitué à la période charnière de la Révolution à l'Empire, époque de métamorphoses. Ce premier rassemblement, lié au mouvement général de constitution des grandes collections publiques à Paris comme en province, coïncide avec les réformes de l'institution médicale libérée du joug des rois et des évêques : à l'École de Santé créée en 1794 succède l'École de Médecine en 1803, qui devient Faculté en 1808. À travers les œuvres qu'il réunit, se mesurent les liens existant à la fois avec le milieu artistique parisien à l'époque néoclassique et avec le centre artistique italien, Rome et la Toscane, alors française. (fig. $\left.\mathbf{n}^{\circ} \mathbf{1 8}\right)$ 


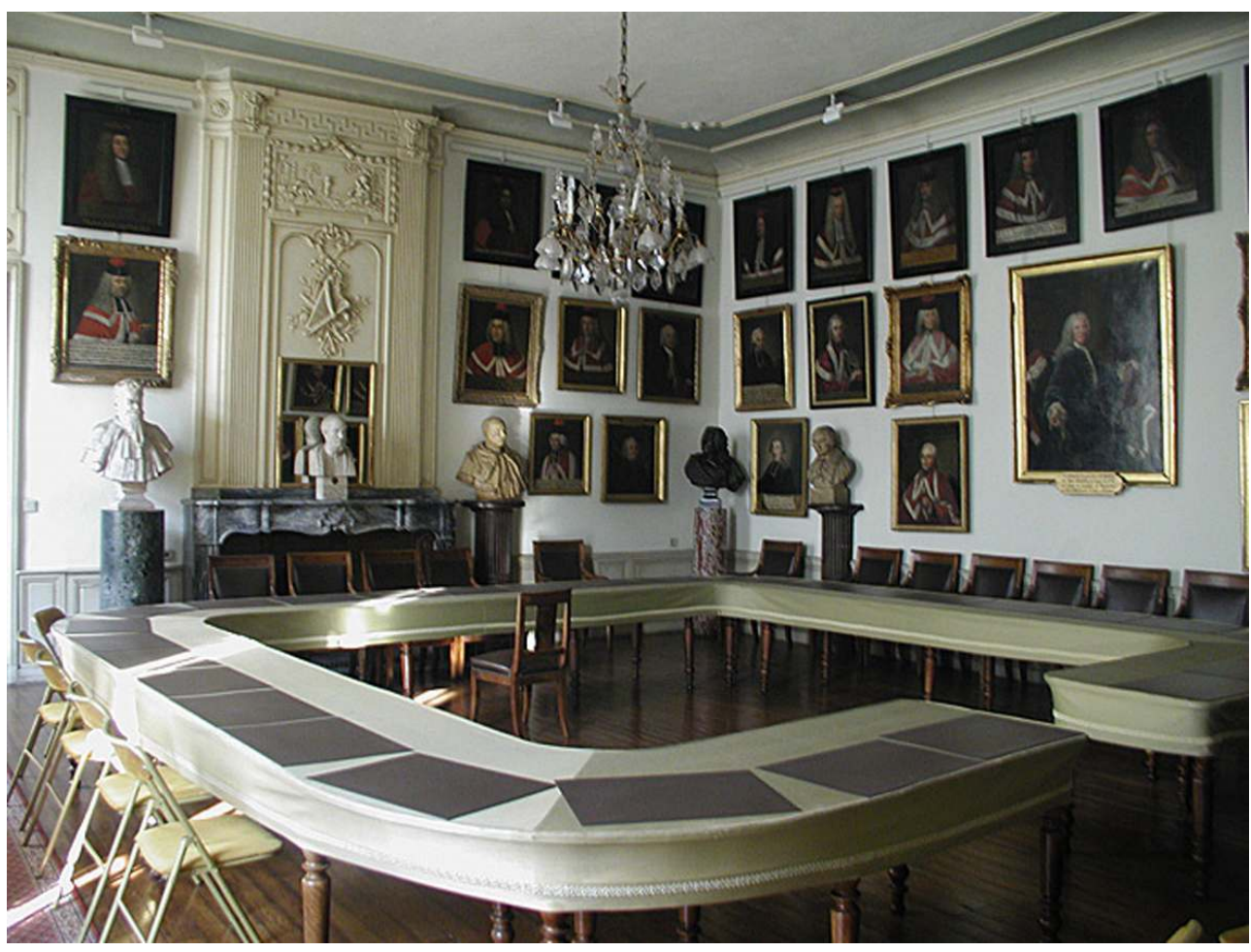

Vue de la salle du Conseil de la faculté de médecine.

(c) DRAC Languedoc-Roussillon.

18 Faisant fi de l'architecte départemental, Chaptal fait appel à Claude-Mathieu Delagardette ${ }^{20}$ (1762-1805), architecte parisien célèbre, primé à l'Académie de France à Rome, pour construire l'Orangerie du Jardin des plantes et l'amphithéâtre d'anatomie. C'est au professeur de médecine légale et d'histoire de la médecine de Montpellier, Gabriel Prunelle (1777-1853), qu'il confie le soin d'inventorier les dépôts littéraires constitués des biens saisis dans les couvents et chez les émigrés, de les répartir à travers la France et d'en faire bénéficier l'École de médecine de Montpellier ${ }^{21}$. Son buste en marbre, envoyé en 1803 pour l'inauguration de l'amphithéâtre d'anatomie, fut réalisé à Carrare par le sculpteur turinois Jean-Baptiste Comolli (1775-1830), élève de Canova. La découverte d'une lettre envoyée par Chaptal éclaire les circonstances de la commande. Son portrait, largement inspiré de celui de Gros a été, selon la tradition, donné par la famille à la faculté. Il fut exécuté après sa mort, en 1832, par un des élèves de Gros, Louis-Joseph Famelli-Semah (1804-1875).

Le 3 juin 1801, Chaptal fait attribuer à l'École de santé de Montpellier un buste antique d'Hippocrate, butin de la campagne d'Italie, acquis par le gouvernement consulaire. Le buste est accompagné d'une inscription éloquente: Olim Coüs nunc Monspelliensis Hippocrates (Jadis de Cos, Hippocrate est aujourd'hui de Montpellier). En 1803, il commande au sculpteur Claude Dejoux (1732-1853), élève de Coustou, deux statues, celle d'Hygie, déesse de la santé, associé à celle de son père Esculape, dieu de la médecine. L'École propose au ministre l'inscription non alibi imponere dii felicius unquam (aucun meilleur endroit pour ces dieux). La même année, il fait réaliser par Jean-Baptiste odiot (1763-1850), orfèvre de Napoléon, la nouvelle masse d'Esculape, emblème de la faculté concédé en 1350 par un privilège du roi comme insigne de la dignité de l'École ${ }^{22}$. Enfin, il 
constitue les premières collections anatomiques en faisant venir, de Florence, les copies tant convoitées des cires anatomiques de Felice Fontana (1730-1805), collection constituée en 1775 pour le grand duc de Toscane Pierre Léopold de Habsbourg-Lorraine, et devant lesquelles Bonaparte était tombé en admiration ${ }^{23}$. (fig. ${ }^{\circ}{ }^{\circ}$ ) $\left(\right.$ (fig. $\left.^{\circ}{ }^{\circ} 20\right)$

Figure 19

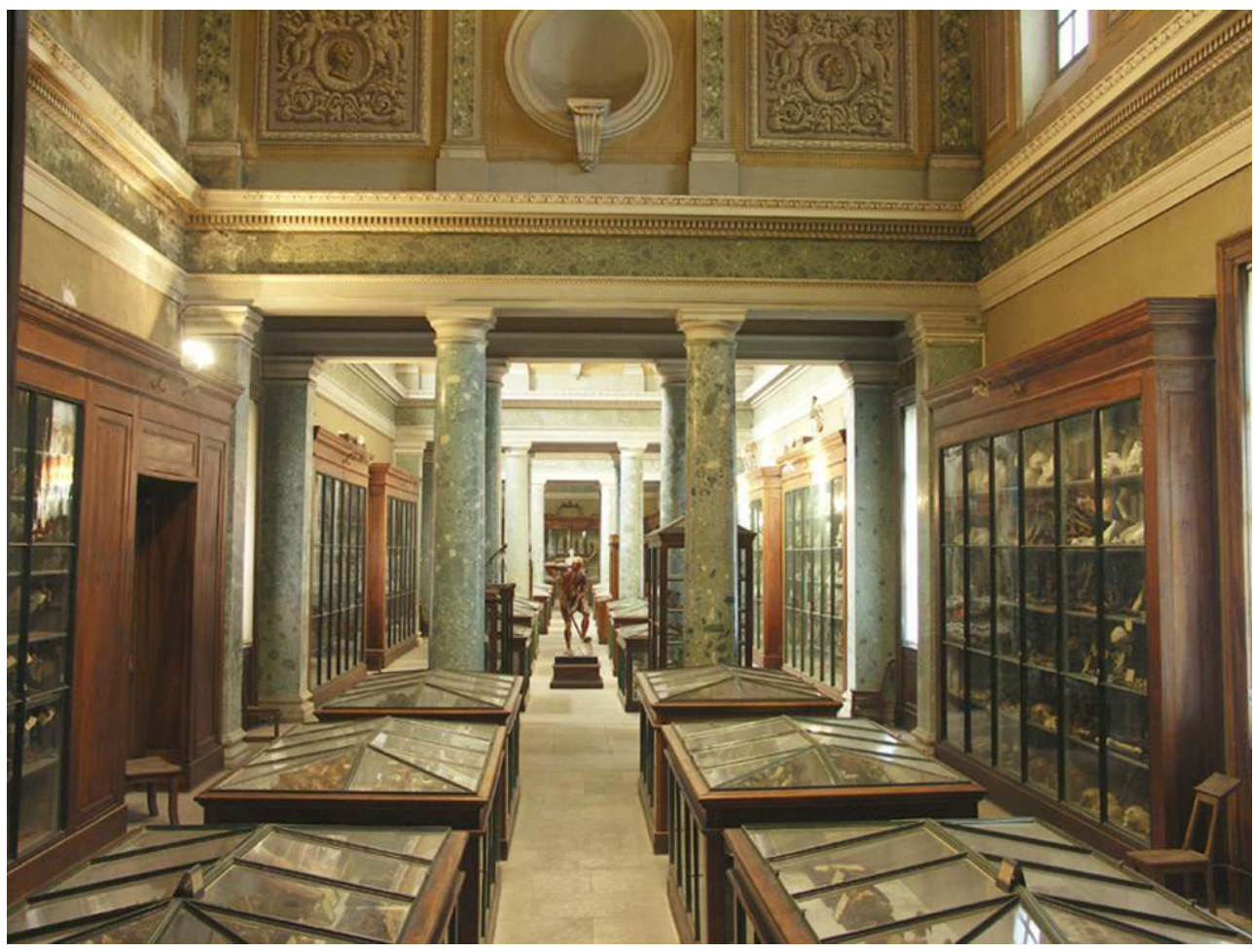

Vue du conservatoire d'anatomie.

(c) DRAC Languedoc-Roussillon. 
Figure 20

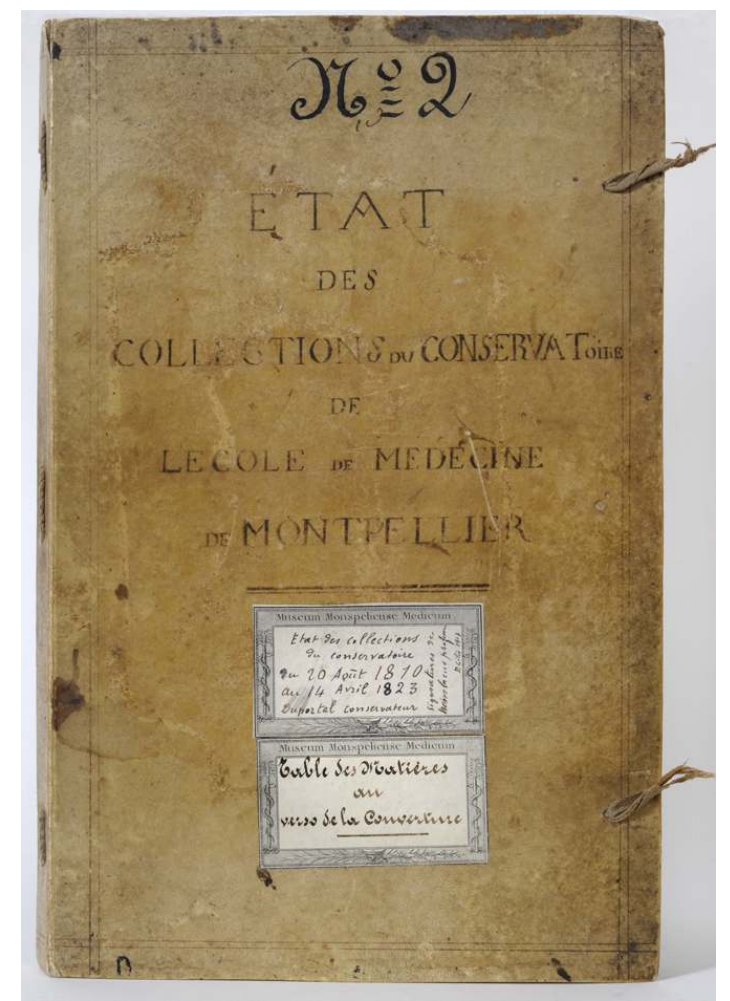

Registre $n^{\circ} 2$ du conservatoire. Q supplément 189 : Registre d'inventaire du conservatoire. État des collections. №2 : 1810 à 1823.

(C) BIU Montpellier.

La protection des collections du conservatoire d'anatomie aurait été impossible sans la découverte en cours d'étude de l'ensemble des neuf registres du conservatoire tenus par les 12 conservateurs successifs, du premier, Virenque, en 1794, au dernier, Gilis, en 1927. Ils répertorient une à une les milliers de pièces: date de leur entrée au musée, provenance, nature, description, numérotation, emplacement dans les vitrines. Ils reflètent aussi l'évolution de la classification. Ces inventaires manuscrits, documents irremplaçables, mémoire du musée, sont de précieuses sources de renseignements à exploiter et font partie du classement.

Héritier des bibliothèques, cabinets d'histoire naturelle ou encore apothicaireries, le conservatoire d'anatomie de Montpellier, créé pour conserver, exposer et exploiter les pièces anatomiques, témoigne d'un même agencement, à la fois symbolique, fonctionnel et décoratif : les volumes sont compartimentés en quatre salles, la taxinomie préside à l'organisation. Temple à la gloire de la médecine, conçu en 1853 par l'architecte PierreCharles Abric (1800-1871), il est décoré par Tommaso Baroffio (1792-1877) et Jean-Pierre Montseret $^{24}$ (1813-1888) de peintures des diverses sciences appliquées à la médecine et des effigies de médecins illustres. Les meubles d'exposition recouvrent les murs dans un ensemble ininterrompu et présentent les milliers de pièces, répertoriées dans les neuf registres du conservatoire, formant une collection d'anatomie normale, pathologique et comparée, d'ostéologie, de tératologie et d'anthropologie : embryons de corps humains conservés dans du formol, momies, squelettes, crânes, écorchés, instruments de chirurgie et planches anatomiques s'ajoutent aux œuvres sculptées, peintes et vernies 
correspondant à deux types de préparation, soit le modèle artificiel en cire, carton pâte, plâtre puis résine, et la préparation naturelle séchée, pièces de dissection dont Honoré Fragonard (1732-1799) fut le meilleur interprète. (fig. $\left.\mathbf{n}^{\circ} \mathbf{2 1}\right)$ (fig. $\mathbf{n}^{\circ} \mathbf{2 2}$ )

\section{Figure 21}

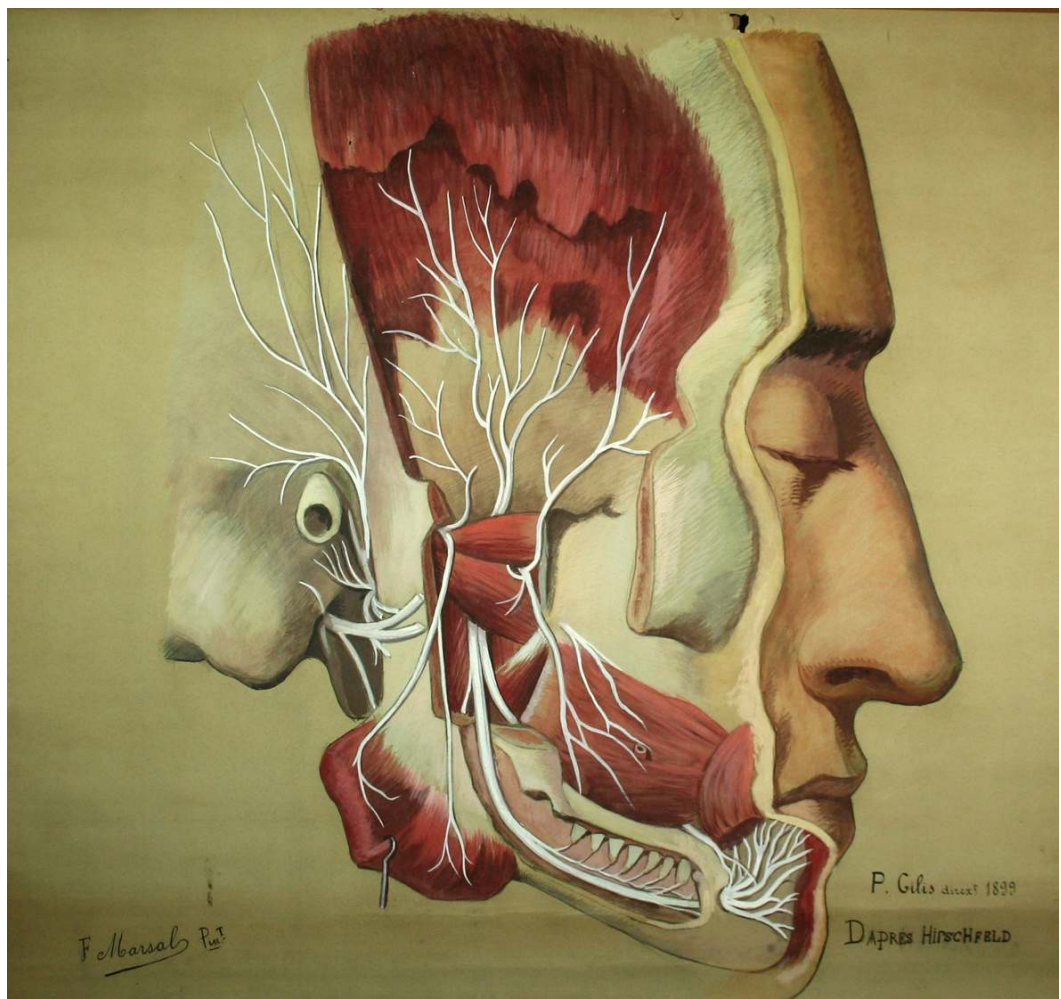

Planche d'anatomie d'après Ludovic Hirschfeld (1814-1876), Paul Gilis, directeur, Édouard Marsal (1845-1929), peintre montpelliérain, 1899.

(c) Marie Conan. 


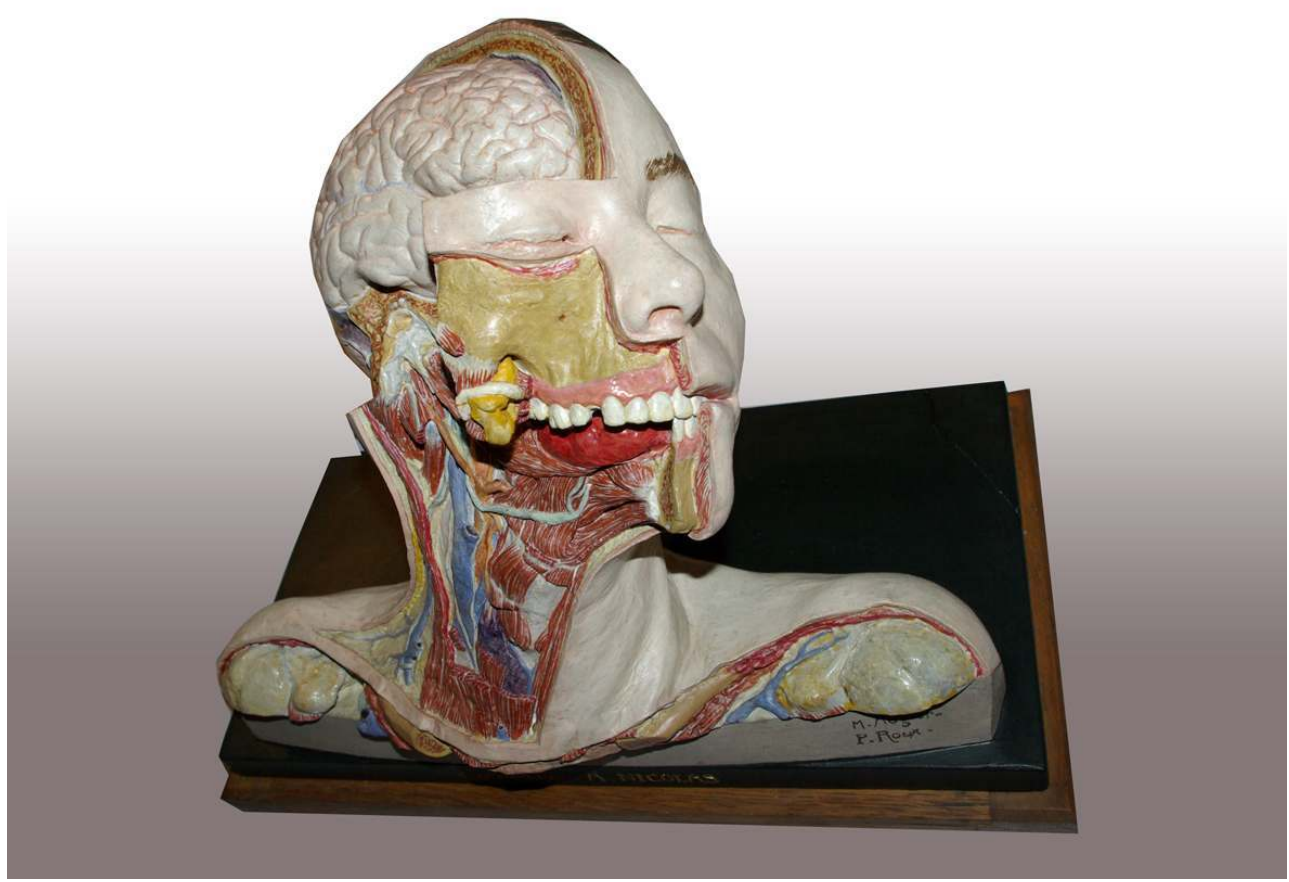

Tête anatomique de M. Augier et P. Roux, collection A. Nicolas, série TC II, plâtre, 20e siècle. (c) Benoît Lafay.

Aujourd'hui, l'anatomie du vivant a supplanté celle du cadavre. Mais les pièces anatomiques, issues des dissections sont toujours là, marquées par les tabous culturels. Si l'anatomie ne règne plus, les sédiments qu'elle a déposés dans la culture sont inscrits dans la mémoire collective et n'ont pas fini d'interroger le réel et le fantastique, le concret et l'imaginaire. Les récentes polémiques autour des foetus découverts à l'hôpital Saint-Vincent de Paul de Paris, la restitution de la Vénus Hottentote d'Afrique du Sud, les expositions de corps plastinés de l'allemand Von Hagens, reposent le problème du statut du corps humain exposé dans les musées, et redonnent toute leur actualité aux pièces anatomiques historiques. L'anatomie est avant tout affaire de regards, une science d'observation, et ses découvertes comme son enseignement reposent sur la pratique, celle des dissections mettant en scène des cadavres, dévoilant les structures internes de l'organisme. Cette mise à jour du caché, cette manipulation de la chair morte a toujours dérangé.

En outre, ces objets à résonances philosophiques, illustration de la pensée scientifique, instruments de connaissance, matériaux d'étude, n'ont plus aujourd'hui la corvée d'être utiles. Ils ne doivent leur rédemption qu'à la continuité de la recherche et de l'enseignement, gage de conservation d'instruments scientifiques devenus obsolètes, et à la pugnacité de quelques "professeurs-patrimonialisateurs». En effet, y compris pour certains scientifiques contemporains, le conservatoire d'anatomie, devenu musée, est un lieu austère, « un musée des horreurs » renfermant les choses d'une histoire dépassée, voire sans histoire utile, et de toute façon désuètes. Si les pièces exceptionnelles, comme celles de la collection Fontana, sont aujourd'hui tombées dans l'oubli, que dire des pièces en série, témoignage d'un siècle ${ }^{25}$ d'enseignement de l'anatomie, mémoire de l'art de la dissection: les centaines de planches anatomiques réalisées par les élèves de Cabanel, 
Marsal, Causse et d'autres artistes comme Suzanne Ballivet, les milliers de préparations naturelles séchées ou obtenues par les techniques d'injection-corrosion, conservées dans des reliquaires de verre et commercialisées par les maisons Tramond ou Auzoux, sont de précieux documents amassés au cours des ans par la succession des chefs des travaux anatomiques, prosecteurs et élèves.

$\mathrm{Au}$ regard des autres collections anatomiques françaises, celle de Montpellier est la plus ancienne et la plus importante conservée dans sa quasi intégralité et dans le lieu créé pour l'accueillir. Elle s'inscrit parmi les autres collections de la faculté, peintures, dessins, estampes et manuscrits, symboles de la tradition humaniste de l'école de médecine de Montpellier. En fonction de son triple intérêt, historique, artistique et scientifique, de la richesse et de la diversité des pièces réunies essentiellement au XIX ${ }^{\mathrm{e}}$ siècle, et bien qu'au niveau national, une sélection sévère s'opère parmi les ensembles repérés, cette collection universitaire d'exception, reflet du dialogue entre art et sciences, mémoire de neuf siècles d'enseignement de la médecine, a été classée en totalité au titre des Monuments historiques en 2004 ainsi que le bâtiment qui l'abrite. À la notion, toute relative, d'œuvre majeure, s'est superposé le caractère d'intérêt public attaché à la conservation du lieu. Le caractère pérenne de la collection étant menacé, la mesure de classement s'imposait d'autant plus comme acte conservatoire ${ }^{26}$.

\section{La collection de moulages de la faculté de Lettres}

Figure 23

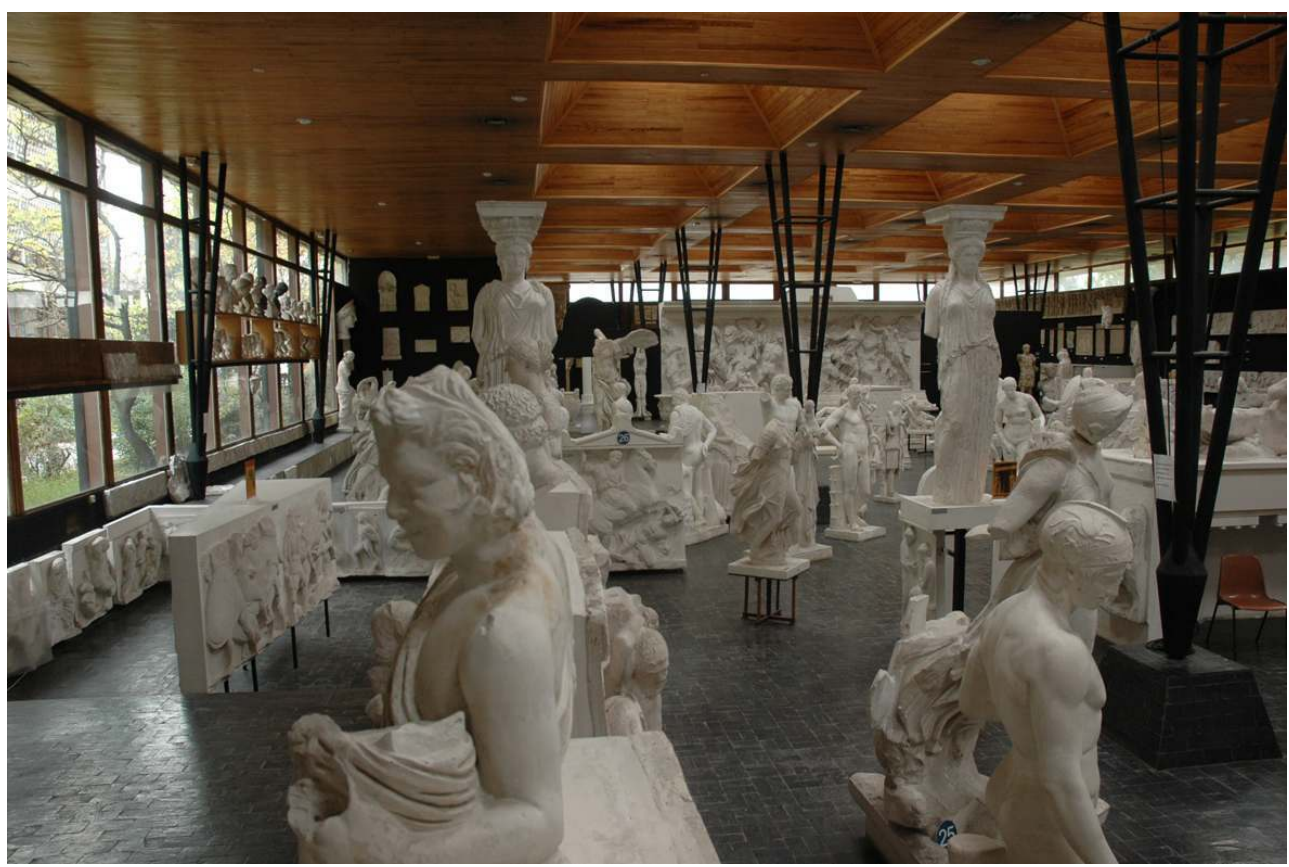

Vue actuelle du musée des moulages.

(c) Denis Gliksman. Université Montpellier III.

Pour un artiste, jusqu'au milieu du XIX ${ }^{\mathrm{e}}$ siècle, le musée des moulages des archétypes antiques est complémentaire du musée d'anatomie des cires et des écorchés. Mais les moulages ont toujours soulevé tempêtes de controverses, tant artistiques que politiques. L'histoire, la genèse et le sort de ces collections de plâtres d'après la sculpture antique et 
médiévale qui ont fleuri dans l'Europe du XIX siècle, se ressemblent un peu partout. Les musées des moulages sont nés de la volonté de quelques grands archéologues et historiens de l'art d'avoir sous les yeux une réplique de qualité des grandes œuvres de la statuaire. Inventorier, collecter, trier, mettre en valeur fut le fait de quelques personnalités particulières, professeurs, savants austères ou amateurs d'art, comme Eugène Müntz en 1889 pour l'École des Beaux-Arts de Paris : appétit d'érudition mis au service des élèves historiens de l'art invités à lire dans l'histoire des sculptures remplacées par leurs substituts de plâtre, les débats entre la France et l'Italie, de l'Antiquité à la Renaissance, entre la doctrine académique et la sensibilité aux arts du passé. Mais la disparition d'une doctrine esthétique, le Beau idéal, pour laquelle l'enseignement s'appuyait sur la copie admise par tous (copies par les prix de Rome jusqu'en 1968), enveloppa le plâtre et le moulage d'un mépris ravageur, dans l'ignorance de sa perspective historico-artistique et sonnant le glas du dépeçage des collections, comme cela fut démontré lors du colloque Moulages de Montpellier en $1997^{27}$. (fig. $\mathbf{n}^{\circ}$ 23)

La Troisième république fut le véritable âge d'or des collections de moulages d'après l'antique, selon le modèle anglais et allemand. En 1860, 120 moulages sont exposés au palais de l'Industrie, à l'image du Crystal Palace. En 1862, 60 moulages sont présentés au Louvre. C'est la défaite de 1870 et la rivalité avec les Allemands qui sont à l'origine de la constitution d'une collection universitaire de moulages d'après l'antique à Strasbourg (1872) qui relance le débat pour la création de collections de plâtre. Les musées parisiens naissent à la fin du XIX siècle : 1873, musée des copies de Charles Blanc au palais des Champs-Élysées ; 1876, Palais des études de l'École des Beaux-Arts de Paris (transféré en grande partie à Versailles en 1970);1879, Musée de sculpture comparée initié par Violletle-Duc ; 1888 musée du Trocadéro ; 1899, musée de Moulages, salle du Manège du Louvre par Félix Ravaisson. Il en est de même pour les collections universitaires, parfois issues des collections des Beaux-arts plus anciennes: 1880, galerie universitaire de Bordeaux (Maxime Collignon); 1890, celle de Montpellier; 1891-96, celle de l'Institut d'art et d'archéologie de Paris (Maxime Collignon et Émile Mâle) ; 1899, collection universitaire de Lyon (Henri Lechat).

27 Selon Emmanuel Schwartz, conservateur des collections de l'École des Beaux-Arts de Paris, «la présentation des moulages survivants se confond avec l'histoire de leur dépérissement. Il n'y a guère que Montpellier qui ait réussi à sauver son musée des moulages». Les musées de Strasbourg et Lyon, dont les collections étaient plus importantes, ont été victimes d'interruptions, dispersions, destructions, mutilations. À Paris, les moulages de l'École des Beaux-Arts sont enlevés en catastrophe en 1970 pour migrer vers les petites écuries de Versailles. La collection dépend du musée du Louvre et Jean-Luc Martinez, chef du département des antiquités grecques, étrusques et romaines, œuvre aujourd'hui à leur restauration pour une présentation accessible au public. Succédant à l'éréthisme du XIX ${ }^{e}$ siècle, la déréliction des collections de moulages se poursuit : déménagées, dispersées, mises en caisse ou évacuées en sous-sol. À Montpellier comme ailleurs, elles sont menacées tant par le désintérêt que par le mauvais état du bâtiment conçu pour la recevoir sur le nouveau campus : des menaces de dispersions ont pesé sur les moulages à partir de 1985, un projet élaboré en 2002, proposa le transfert de la collection à la chapelle Saint-Charles. À la demande de l'université et dans le souci de préserver un des derniers témoins de ces grands ensembles, la collection de moulages de Montpellier a été classée en totalité en $2009^{28}$. 
La protection au titre des Monuments historiques : un outil de conservation opérationnel

Figure 24

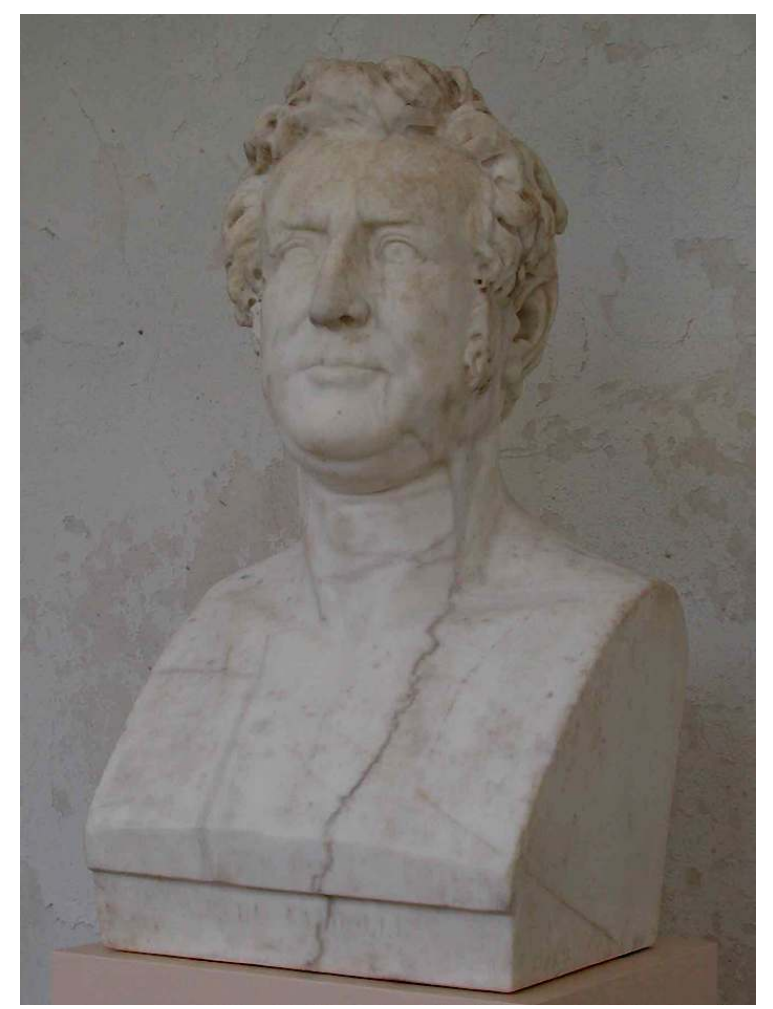

Buste en marbre d'Augustin Pyrame de Candolle au Jardin des Plantes, Antoine Custor, 1878. (c) Benoît Lafay, restaurateur du patrimoine. 


\section{Figure 25}

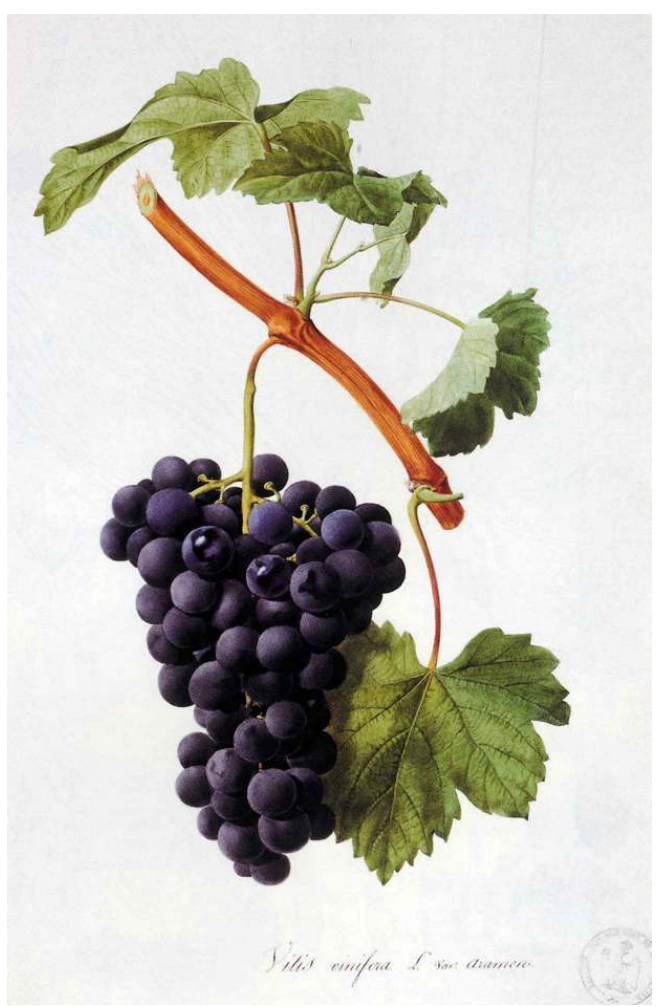

Vélins de la collection Node-Véran (1773-1852).

(c) Service des Collections, Montpellier II. 


\section{Figure 26}

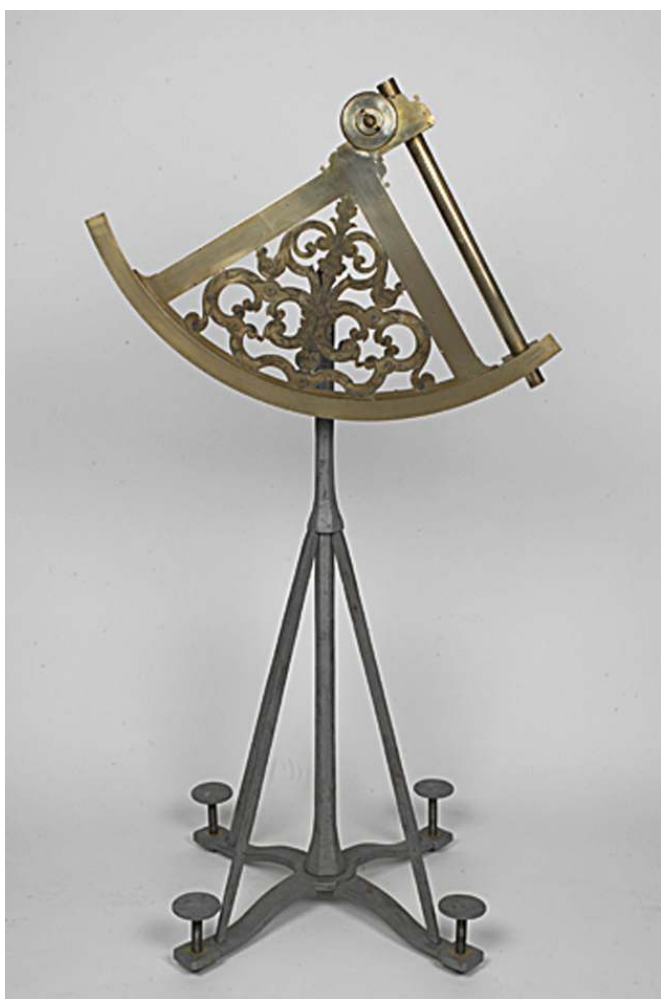

Quart de cercle mobile Cadot, 1730. Ancienne collection de l'Observatoire d'astronomie. (C) Service des Collections, Montpellier II. 
Figure 27

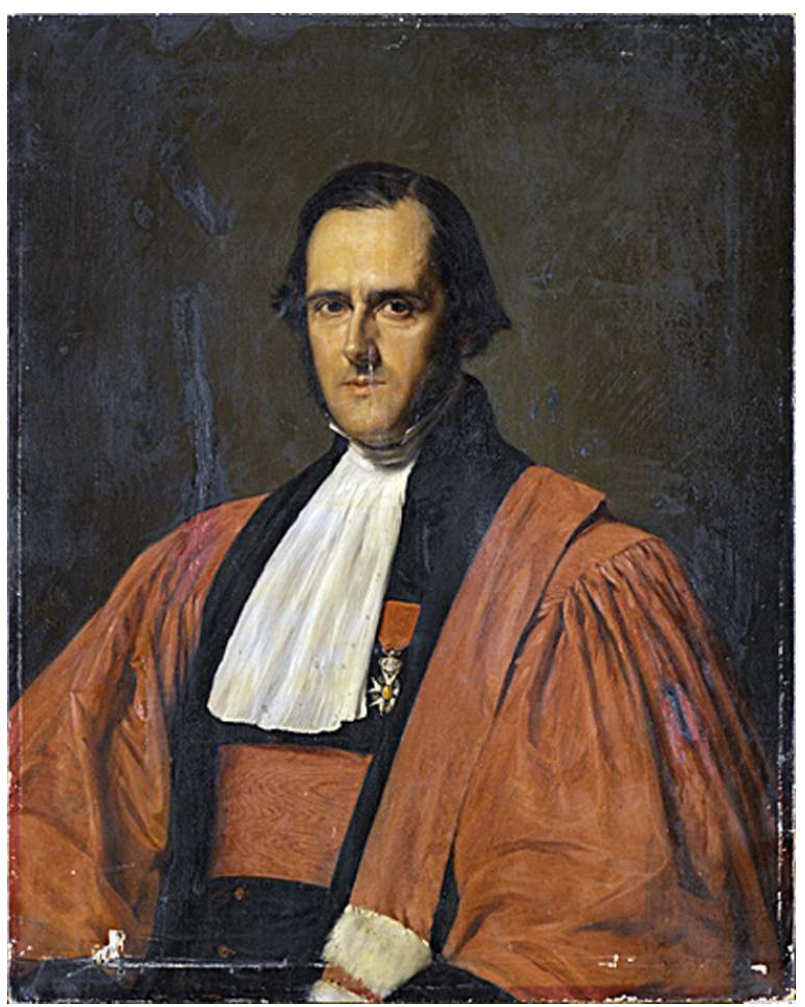

Portrait de l'astronome Édourd-Albert Roche (1820- 1883), Charles Matet, 1875.

(c) Service des Collections, Montpellier II.

L'étude globale du patrimoine universitaire a permis de classer " massivement » au cours de ces cinq dernières années, les collections des Universités Montpellier I, II et III, désormais clairement identifiées, par le classement ou l'inscription. Dans ce contexte, ne pas protéger tel ou tel objet ou collection à la valeur historique, artistique ou scientifique démontrée aurait été le dévaloriser au regard des autres, notamment dans le cadre de la préfiguration d'un pôle patrimoine historique que devraient construire les partenaires du projet Campus faisant de Montpellier l'un des dix centres universitaires internationaux de France. En fonction des demandes successives des trois universités et au fil des commissions nationales des Monuments historiques, ont été classés: la collection d'anatomie, soit 5688 pièces le 5 octobre 2004, la galerie de 230 portraits de professeurs le 20 mai 2005 (Montpellier I), plus de 200 éléments provenant de l'ancien Observatoire dont 50 instruments d'astronomie et 5 portraits d'astronomes le 19 janvier 2006 (Montpellier II), la collection de moulages (553 sculptures, soit 718 éléments individuels) le 15 janvier 2009 (Montpellier III) et 22 portraits sculptés de la faculté de médecine le 28 juillet 2009 (Montpellier I). Les herbiers des XVII et XVIII ${ }^{e}$ siècles de l'Institut de botanique et la collection de 978 vélins en 10 volumes de Toussaint-François Node-Véran (1773-1852), créée en 1808 par Augustin Pyrame de Candolle et conservés par l'Université de Montpellier II sont en cours de classement. De nombreuses pièces ont également été inscrites : 35 portraits, tableaux et meubles, un écorché d'Honoré Fragonard, 118 pièces de la collection ethnologique de la faculté des sciences, 4 tableaux et un portrait sculpté du laboratoire de recherche de la Station de biologie marine de Sète (Montpellier II), 34 portraits sculptés, 13 dessins anatomiques de Camille Descossy, 23 tables de dissection de la faculté de médecine, 33 portraits peints et les collections du droguier de la faculté de 
pharmacie (10 000 échantillons), deuxième droguier de France par son importance (conservé par l'Université de Montpellier I). Les œuvres de l'ancien palais universitaire, actuellement Rectorat, sont en cours de protection. (fig. $\left.\mathbf{n}^{\circ} \mathbf{2 4}\right)$ (fig. $\left.\mathbf{n}^{\circ} \mathbf{2 5}\right)\left(\right.$ fig. $\left.\mathbf{n}^{\circ} \mathbf{2 6}\right)($ fig. $\left.n^{\circ} 27\right)$

La protection Monument historique est un outil opérationnel, scientifiquement et méthodologiquement reconnu et demandé par les autorités universitaires. Le classement, par la reconnaissance qu'il implique, est en soi un outil de conservation. Mais il n'aurait aucun sens, s'il n'était suivi d'un plan de restauration préalable à l'ouverture au public des collections. Sauvegarder le patrimoine historique dans une université n'est pas chose aisée. Il encombre, et les budgets réduits laissent souvent peu d'espoir pour un investissement massif à l'échelle des collections. Le partenariat noué en 2005 entre la DRAC et l'université pour la sauvegarde du patrimoine universitaire est exemplaire, car malgré l'émergence ces vingt dernières années de l'intérêt pour le patrimoine scientifique, une telle démarche n'a rien d'évident aujourd'hui, elle est même unique en France $^{29}$. Il serait pourtant vain de penser que la protection du patrimoine pourrait, même en théorie, suppléer à l'effort collectif de gestion qui s'impose pour l'université. Comme le soulignait André Chastel, «le patrimoine se reconnaît au fait que sa perte constitue un sacrifice et que sa conservation suppose des sacrifices ${ }^{30} »$. (fig. $\mathbf{n}^{\circ} \mathbf{2 8}$ )

Figure 28

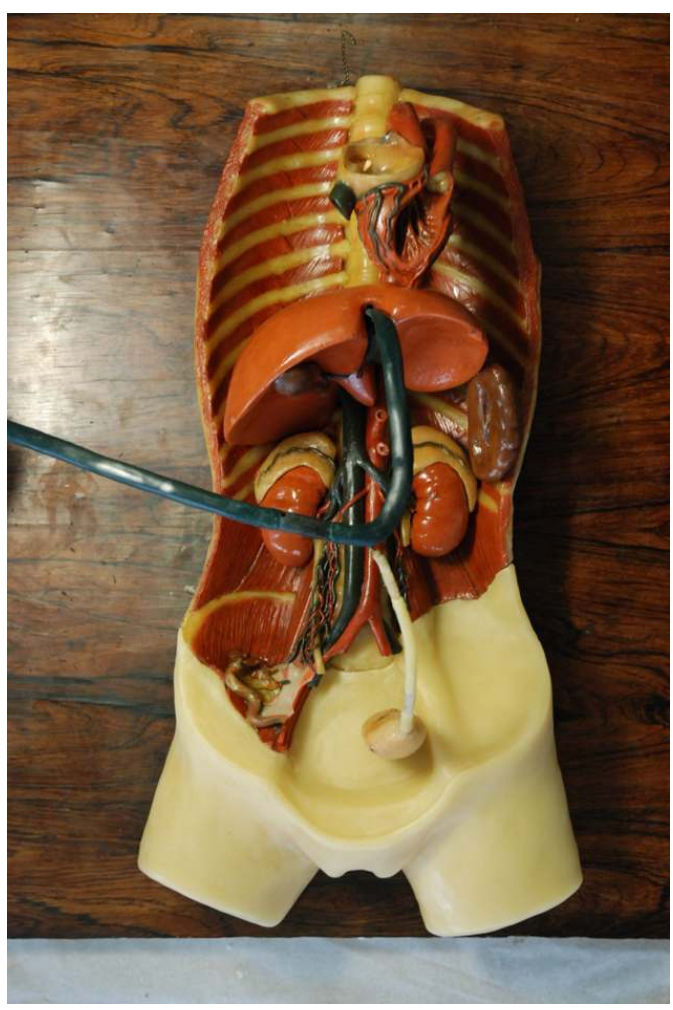

Restauration d'une cire anatomique de Felice Fontana par Isabelle Pradier.

(c) Isabelle Pradier, restauratrice du patrimoine.

Pour le Musée d'anatomie, les études préalables ont conduit à la formation d'un réseau d'intervenants et les institutions spécialisées, centres de recherche, de restauration et musées ont été sollicités: Centre interrégional de conservation restauration du patrimoine de Marseille (CICRP) ${ }^{31}$, Centre de conservation restauration des Musées de 
France (C2RMF) ${ }^{32}$, Laboratoire de recherche des monuments historiques (LRMH), Musée Fragonard de l'École vétérinaire de Maisons Alfort ${ }^{33}$, Musée de l'Homme ${ }^{34}$, Muséum national d'histoire naturelle à Paris ${ }^{35}$, Musée de La Specola et Centre de restauration Opificio delle Pietre Dure ${ }^{36}$ à Florence. Grâce à ces collaborations, la réalisation d'une étude sur la sûreté des œuvres et d'un bilan de l'état de conservation des collections a permis de définir un schéma d'opération de rénovation globale ${ }^{37}$. Suite aux expertises dans les différents domaines concernés, les travaux de conservation-restauration sont mis en œuvre progressivement, sur de petites entités, en rapport avec les moyens actuels ${ }^{38}:$ cires de Fontana ${ }^{39}$, planches anatomiques ${ }^{40}$, écorchés ${ }^{41}$, bocaux de tératologie ${ }^{42}$. Enfin, un projet d'études par imagerie médicale est en cours ${ }^{43}$. En 2008, un partenariat avec le Conseil général de l'Hérault ${ }^{44}$ permet de préparer un film destiné à préserver la mémoire du lieu et des différentes phases de restauration. En 2010, une étude des momies par imagerie médicale est réalisée par le CHRU de Montpellier et le musée du Louvre sous l'égide de Samuel Mérigeaud ${ }^{45}$. La même année est confiée à Isabelle Pradier la restauration d'une cire anatomique de Felice Fontana dans le cadre de son diplôme de restauratrice de $l^{\prime}$ Institut national du patrimoine ${ }^{46}$. (fig. $\mathbf{n}^{\circ} \mathbf{2 9}$ )

Figure 29

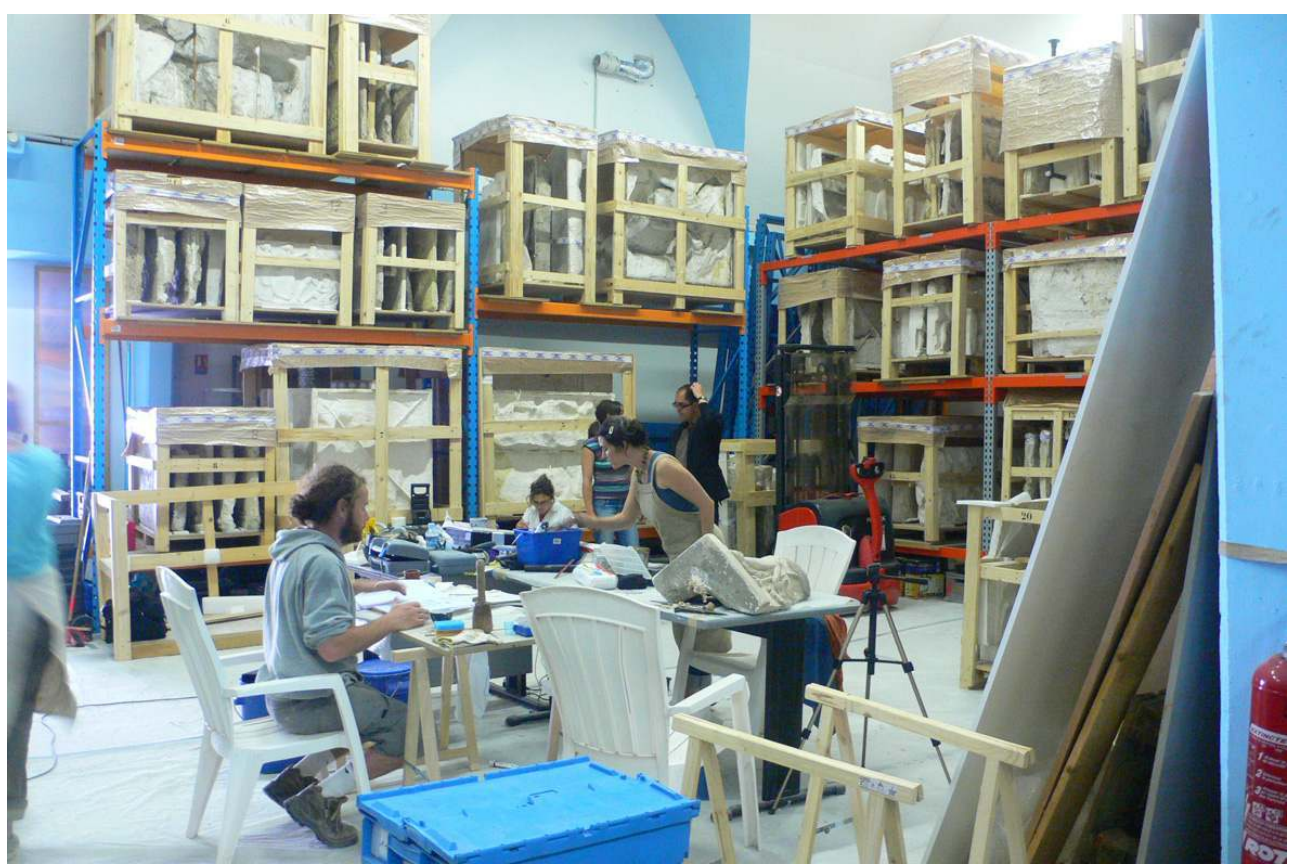

Chantier de restauration des moulages par l'équipe de Pascale Roumegoux.

(c) Hélène Palouzié.

Le classement de la collection du Musée des moulages a permis de redéfinir le projet de réhabilitation du musée, afin de conserver toute la cohérence de présentation de cette collection patrimoniale, de créer un comité de pilotage et d'effectuer les études préalables au chantier des collections. La rénovation du bâtiment ayant été conçue sans tenir compte des collections (aucune mesure de protection des œuvres n'avait été alors envisagée), la DRAC a recommandé les expertises de MM. Martinez (Musée du Louvre), Roch Payet (Institut national du Patrimoine), et Berthelot (CNRS/Ministère de la Culture), afin de faire évoluer le projet. La fragilité des moulages en plâtre, l'état général d'empoussièrement des œuvres, les modes de fixation défectueux pour l'intégrité de 
certaines pièces, l'impossibilité de déplacement de certains éléments, l'état particulièrement critique de certains moulages altérés par la pluie et les inondations, ont conduit à définir une méthodologie adaptée à la conservation des œuvres avant les travaux sur le bâtiment: inventaire informatisé ${ }^{47}$, relevés topographiques et panoramiques pour la traçabilitée ${ }^{48}$, bilan sanitaire et marquage des œuvres ${ }^{49}$. Comme pour le musée d'anatomie, un film a été commencé, avec la captation d'images avant le déménagement et l'interview des professeurs liés à l'histoire de la collection. Le bilan sanitaire intégré à la base de gestion des moulages a permis d'élaborer le cahier des charges pour la $1^{\text {ère }}$ tranche d'opérations sur les collections, inhérente à la rénovation du bâtiment. Cette phase, programmée en 2009, comprenant la conservation préventive, le déménagement et stockage ainsi que la restauration des pièces les plus altérées, s'est achevée en juin $2010^{50}$. La seconde phase concerne la muséographie et la repose des œuvres. Elle est en cours d'élaboration par Rosa Plana et Jean-Luc Martinez.

Pour ces objets de mémoire immobilisés, deux logiques se complètent: la logique de sauvegarde in situ initiée par le classement Monument historique et la logique de présentation muséale. Le classement Monument historique a été un levier indispensable pour la sauvegarde des collections et l'université multiplie aujourd'hui des actions de valorisation, de médiatisation et d'ouverture au public de son patrimoine. Son objectif est, à terme, la gestion des collections selon les modalités définies par le label musée de France.

\section{NOTES}

1. - Ces pages sont le développement d'un précédent article « Les collections universitaires de Montpellier : la protection Monument historique, un outil de conservation ». Monumental, 2011, p. 24-26. DELMAS, Paul. Le patrimoine de la faculté de médecine de Montpellier. Montpellier, 1921, p. 5. 2. - FRANÇOIS, Michèle. Jean Balladur et la Grande-Motte. L'architecte d'une ville. Collection DUO, DRAC/CRMH, 2010.

3. - Icônes et Idoles, regards sur l'objet Monument Historique. PALOUZIÉ, Hélène (dir.). Arles : Actes Sud, 2008, p. 41-54. POISSON, Olivier. «L'objet Monument historique ». Dans PALOUZIÉ, Hélène (dir.). Arles : Actes Sud, 2008.,

4. - PALOUZIÉ, Hélène. "Icônes et Idoles, regards sur l'objet Monument Historique ». Dans PALOUZIÉ, Hélène (dir.). Arles : Actes Sud, 2008, p. 316-317 et 320-323.

5. - POULOT, Dominique. «Le ministre de l'Intérieur : la fondation des musées de province». Dans PERONNET, Michel. Jean-Antoine Chaptal (1756-1832). Actes du congrès national des sociétés savantes de Montpellier, faculté de médecine, 1985. Montpellier, 1986, p.162-176. POULOT, Dominique. Patrimoine et musées. L'institution de la culture. Paris : Hachette, 2001, p. 66-76.

6. - DULIEU, Louis. «Les portraits des universitaires montpelliérains à travers les galeries de tableaux ». Bulletin de l'Académie des Sciences et Lettres de Montpellier, 1996, nouvelle série, tome 27, p. 301-310. DULIEU, Louis. «Un trésor méconnu: la galerie des tableaux de l'hôpital de Montpellier ». Dans DUMAS, Robert (dir). Histoire de l'hôpital général et l'hôpital Saint-Charles de Montpellier. Montpellier : Sauramps médical, 2002. 
7. - Je remercie Hélène Lorblanchet, conservatrice de la bibliothèque de la faculté de médecine de Montpellier et Flore César, conservatrice déléguée des antiquités et objets d'art de l'Hérault pour leur contribution à la redécouverte de ces tableaux.

8. - La similitude entre la situation actuelle de l'université de Montpellier et celle de la Troisième république est soulignée dans l'article de: LAURENS, Jean-Paul. Aperçu historique sur l'interdisciplinarité à Montpellier. Communication pour KYKLOS le 2 février 2010. kyklos.asso.fr/ index.php/download_file/-/view/18/. En 1289, une université unique regroupe le droit, la médecine et les arts libéraux : mais dans les faits l'université est bicéphale : d'un côté l'université de médecine, de l'autre l'université générale. À l'université d'Ancien Régime, succèdent progressivement 3 facultés : en 1794 création de l'École de santé qui deviendra École de médecine en 1803 ; en 1803 création de l'École de pharmacie; en 1808 l'École de médecine devient faculté de médecine et sont créées la faculté de sciences et la faculté de lettres (qui disparaîtra en 1816 et ne réouvrira qu'en 1838). La cérémonie d'inauguration des facultés en 1810 célèbre donc trois facultés : médecine, sciences et lettres. La faculté de droit ne verra le jour qu'en 1878.

9. - Ernest Michel (1833-1902), élève de Charles Matet, puis d'Alexandre Cabanel, ${ }^{\text {er }}$ prix de Rome en 1860, fut conservateur du musée Fabre de 1871 à 1902 et directeur de l'École des Beaux-Arts de Montpellier.

10. - Michel Maximilien Leenhardt (1853-1941), élève en 1871 d'Ernest Michel à l'École des BeauxArts de Montpellier, puis en 1874 d'Alexandre Cabanel à l'École des Beaux-Arts de Paris, participe dès 1879 au Salon des Artistes Français, dont il sera plusieurs fois lauréat avant d'en devenir sociétaire en 1883.

11. - Édouard Marsal (1845-1929), élève aussi de Matet et Cabanel, professeur de dessin, a laissé une œuvre d'illustrateur considérable; à la faculté de médecine, il a relevé des décors de la chapelle des Évêques devenue salle des Actes et réalisé de nombreuses planches d'anatomies du conservatoire. Ses enfants, Fernand, Louis et Suzanne, peintres également, seront associés à ses travaux.

12. - DELMAS, Paul. Le patrimoine de la faculté de médecine de Montpellier. Montpellier, 1921, p. 13.

13. - Gustave Charles Bonaventure Chancel (1822- 5 août 1890), professeur de chimie à la faculté des Sciences (nombreux travaux appliquant la chimie à l'œnologie : plâtrage des vins, coloration artificielle...), doyen de cette faculté de 1865 à 1879, puis recteur de l'académie de Montpellier de 1879 à 1890, a fortement marqué son université d'adoption, dont il fêta le VIe centenaire. C'est sous son décanat qu'a été décidé le transfert des facultés des Sciences, Lettres et Droit dans l'ancien hôpital Saint-Éloi.

14. - Ferdinand Castets (1838-1911), doyen de la Faculté des Lettres de 1881 à 1902, réorganise l'enseignement en instaurant les chaires d'histoire, de grammaire et de littérature comparée, d'archéologie, de paléographie et dote la Faculté d'un musée de moulages. Conseiller municipal de Montpellier de 1881 à 1904, il est maire de la ville de 1893 à 1896. Il est aussi membre de l'Académie des sciences et des Lettres dès 1887 et de la société archéologique de Montpellier.

15. - ROUZAUD, Henri. Montpellier 1890. Les fêtes du VI centenaire de l'Université. Montpellier: Camille Coulet, 1891. Journal l'Université de Montpellier, 1890 (18 numéros); numéro spécial Le Centenaire. Montpellier : Firmin et Mantane, 1890.

16. - Lettre du ministre de l'Instruction publique et des Beaux-Arts à Chancel, Recteur de l'Académie de Montpellier, Paris, $1^{\mathrm{er}}$ février 1889. Archives départementales de l'Hérault, fonds de la Préfecture, 1 T 2231.

17. - ROUZAUD, Henri. Montpellier 1890. Les fêtes du VIe centenaire de l'Université. Montpellier : Camille Coulet, 1891, p. 53-54.

18. - Charles Flahault (1852-1935), illustre représentant de l'histoire de la botanique, fut pionnier dans de nombreux domaines, cartographie botanique, phytogéographie, phytosociologie et écologie forestière. D'abord modeste jardinier au Muséum d'histoire naturelle de Paris, il est 
nommé professeur en 1883 à la Faculté des sciences de Montpellier avant de créer l'Institut de botanique, dont il sera le premier directeur.

19. - Création par la République le 4 décembre 1794 des trois Écoles de Santé : Montpellier, Paris et Strasbourg. En 1795, Chaptal est nommé professeur de chimie appliquée aux arts et à l'industrie à l'École de santé de Montpellier et a pour mission de veiller à sa réorganisation. Il fut aussi l'artisan de la création des musées de province (loi Chaptal du $1^{\mathrm{er}}$ septembre 1801) dont celui de Montpellier.

20. - VERDIER, Thierry. «Une architecture républicaine, l'orangerie du Jardin des Plantes de Montpellier ». Annales historiques de la Révolution française, n³09, 1997, p. 441-450.

21. - Plus d'un millier de volumes, dont deux tiers antérieurs à l'imprimerie, ont été attribués à la faculté de médecine de Montpellier. LORBLANCHET, Hélène, VIAL, Mireille. «La bibliothèque universitaire de médecine». Médecine, art et histoire à Montpellier. Nunc Monspeliensis Hippocrates, Hors série, Sauramps médical, 2002, p. 14-49.

22. - Cette masse est à mettre en relation avec le sceptre de la faculté des Sciences récemment retrouvé par le service des collections de Montpellier II (Flore César) et que j'ai pu identifier par les poinçons comme étant une œuvre du célèbre orfèvre Montpelliérain, Placide Boué, datant des années 1809-1819.

23. - PALOUZIÉ, Hélène. Felice Fontana et l'aventure des cires anatomiques de Florence à Montpellier. Collection Duo, dirigée par Delphine Christophe, Conservatrice régionale des monuments historiques, DRAC Languedoc-Roussillon, $\mathrm{n}^{\circ} 1$, septembre 2010.

24. - Tommaso Baroffio (1792-1877) : peintre décorateur suisse italien établi à Marseille, conseillé par Réattu à Fabre pour le décor de l'hôtel de Massilian (actuel musée Fabre). Jean-Pierre Montseret (1813-1888) : portraitiste de la société montpelliéraine. Ils s'allient aussi pour le décor de l'église Saint-Denis de Montpellier. Voir: PALOUZIÉ, Hélène. «Les œuvres d'art de l'église Saint-Denis ». Saint-Denis de Montpellier, genèse et évolution d'une paroisse. Montpellier : éditions de l'Espérou, 2008, p. 74-107.

25. - PALOUZIÉ, Hélène. Felice Fontana. L'aventure des cires anatomiques de Florence à Montpellier. Collection DUO, DRAC/CRMH, 2011.

26. - «Les musées Universitaires ». Museum international. Éditions Unesco, n²06 et 207, 2000. LOURENÇO, Marta C. « Musées et collections des universités : les origines ». La Revue musée des arts et métiers, 2004, $\mathrm{n}^{\circ} 41$, p. 51-61.

27. - Moulages. Actes des rencontres internationales sur les moulages, Montpellier, 14-17 février 1997. Montpellier : Université Paul-Valéry, 1999. De nombreux colloques et ouvrages ont traité le sujet, notamment: Le moulage. Actes du colloque international, 10-12 avril 1987. Paris: La Documentation française, 1988 ; Les moulages de sculptures antiques et l'histoire de l'archéologie. Actes du colloque international, 24 octobre 1997, Paris: Hautes études du monde romain. Genève, 2000 ; Le plâtre, l'art et la matière. Colloque, octobre 2000, Cergy-Pontoise. Paris, 2001 ; PINATEL, Christiane. "Origines de la collection des moulages d'antiques de l'École nationale des BeauxArts de Paris ». Dans LAURENS, Annie-France et POMIAN, Krzystof (dir.). L'Anticomanie - La collection d'antiquités aux $18^{e}$ et $19^{e}$ siècles. Paris, 1992, p. 307-325 ; CUZIN, J.-P., GABORIT, J.-R., PASQUIER, A. D'après l'antique (cat. d'exp.). Paris, 2000 ; MARTINEZ, Jean-Luc. « Les moulages en plâtre d'après l'antique au musée du Louvre : une utopie du XIX siècle ». Les antiques du musée Napoléon. Paris : R.M.N., 2004, p. 78-82 ; PICARD-CAJAN, Pascale. Ingres et l'Antique (cat. exp.). Arles : éd. Actes Sud, 2006.

28. - Je remercie M. Jean-Luc Martinez, chef du département des antiquités grecques, étrusques et romaines du Louvre, et M. Lorenz Baumer, professeur d'histoire de l'art antique, professeur associé à l'université Paul Valéry pour la défense du dossier lors de la Commission nationale des Monuments historiques du 25 novembre 2008. 
29. - Ce partenariat a été engagé par Didier Deschamps, directeur régional des Affaires culturelles et Robert Jourdan, conservateur régional des Monuments historiques avec la présidente de l'université Montpellier I, Madame Dominique Deville de Périere.

30. - Extrait du film sur André Chastel et l'Italie "Un sentiment de bonheur », 1990, réalisé par Edgardo Cozarinsky, <http://films.louvre.fr/realisateurs/c/cozarinsky-edgardo.html>, les Films d'ici/musée du Louvre.

31. - 2005 : Expertise de la collection par Élisabeth Mognetti (CICRP) ; 2008 : Expertise de Roland May (CICRP) ; 2009: Mission d'étude scientifique des cires de Fontana par Nicolas Bouillon (CICRP).

32. - 2008: Expertise pour la conservation des momies par Marie-Hélène Rutschowscaya (Département des antiquités égyptiennes du musée du Louvre), et Hélène Guichard (conservateur au C2RMF).

33. - 2008: Expertise des écorchés par le professeur d'anatomie Christophe Degueurce, conservateur du musée de l'École Vétérinaire d'Alfort (MéVA - ancien musée Fragonard) et Laure Cadot (restauratrice, Centre de recherche et de restauration des musées de France pour le dossier « restes humains »).

34. - 2009 : Expertise d'Anne Raggi (Musée de l'Homme) sur les pièces anatomiques conservées dans les liquides.

35. - 2010 : Expertise de Marc Herbin, chargé des collections conservées dans des fluides du Muséum national d'histoire naturelle.

36. - 2007 : Conseils en restauration des cires Fontana par l'Opificio delle Pietre Dure e Laboratori di Restauro et le musée de la Specola.

37. - Avril 2006/mars 2007 : Étude en conservation des collections par la société LP3. 2007 : étude de sûreté réalisée par les commandants Cadias et Théfo de la mission sûreté du patrimoine, puis étude de la société Sipal.

38. - Sur cinq années, le coût global de conservation des collections, financé à parts égales par la DRAC et l'Université est de 200000 euros.

39. - 2007-2009 : Restauration des cires de Fontana par Laurence Chicoineau.

40. - 2009-2010 : Conservation/restauration de 500 planches anatomiques par l'équipe réunie par Danielle Amoroso, Marie Connan, Armelle Demongeot et Marina Weissman.

41. - 2009 : Étude en conservation/restauration de l'écorché de Delmas par Laure Cadot.

42. - 2010: Restauration des bocaux de tératologie par Frank Meyer et Jean-Marc Gory du laboratoire d'anatomie sous la direction de M. Bonnel, professeur d'anatomie, chargé du conservatoire, et de M. Herbin.

43. - 2010 : Convention avec le CHU de Montpellier pour la documentation des restes humains et œuvres d'art par imagerie médicale par Samuel Mérigeaud.

44. - La convention entre la DRAC et le Conseil général de l'Hérault sur les lieux de mémoire permet avec le cinéaste Luc Bazin de réaliser des films sur les collections universitaires, le Jardin des plantes, le conservatoire d'anatomie et musée des moulages. Celui consacré au Jardin des plantes est en voie d'achèvement.

45. - 2010 : convention avec le CHU de Montpellier pour la documentation des restes humains et œuvres d'art par imagerie médicale par Samuel Mérigeaud.

46. - L'Institut national du Patrimoine et la DRAC Languedoc-Roussillon organisent à Montpellier en novembre 2012 un colloque européen sur les collections anatomiques.

47. - Il a été réalisé par l'équipe universitaire, transposé et complété en 2007 par la Conservation régionale des monuments historiques sur la Base Objet de la DRAC. Cette base réunit les données historiques, techniques et photographiques des œuvres.

48. - En 2008-2009, des plans de situation des œuvres et relevés topographiques ont été réalisés par l'École d'architecture de Montpellier. Des panoramiques et « fresques murales » effectués par 
Denis Gliksman, en lien avec Michel Berthelot, permettent de garder la mémoire de l'emplacement des sculptures.

49. - Il a été réalisé en 2008-2009 par Pascale Roumegoux et Anthony Quatreveau sur l'ensemble de la collection. Il comprend, pour chacun des 718 éléments, un descriptif technique et l'état sanitaire (constat d'état avec photos des altérations particulières), et prend également en compte les contraintes liées à la manipulation avant déplacement, à la dépose, au stockage (in situ avec déplacement ou coffrage, lieu de stockage extérieur) et à la repose. Sont aussi mentionnées les restaurations à exécuter en priorité. Ces fiches sanitaires ont été enregistrées sur la base de données, couplées avec les fiches d'inventaire des œuvres.

50. - La première phase de restauration des moulages, d'un coût global de 84000 euros, a été financée à parts égales par la DRAC et l'Université. Elle concernait la restauration de 163 reliefs en plâtre. L'opération réalisée par une équipe de huit restaurateurs dirigée par Pascale Roumegoux s'est déroulée en juin 2010 dans les locaux de la société Gaby à Nîmes. Voir : PALOUZIÉ, Hélène. "Conservation/restauration de la collection de moulages ». La faculté de Lettres de Montpellier, Collection DUO, DRAC/CRMH, 2012 (à paraître).

\section{RÉSUMÉS}

Face aux difficultés de conservation de son patrimoine historique, les autorités universitaires de Montpellier ont demandé sa protection au titre des Monuments historiques. Outil de conservation fédérateur et reconnu par tous, il s'est avéré être un levier indispensable à la sauvegarde des bâtiments historiques et des collections qu'ils contiennent.

Facing the difficulty of preserving its heritage, the Montpellier University authorities required its Historic Monument filling. This federative conservation tool is recognized by everyone. It is a $\mathrm{n}$ indispensable way of safeguarding historical buildings as well as their collections.

\section{INDEX}

Mots-clés : anatomie, collections scientifiques, Monuments historiques, moulage en plâtre, restauration, université

\section{AUTEUR}

\section{HÉLÈNE PALOUZIÉ}

Conservatrice à la conservation régionale des monuments historiques, Direction régionale des affaires culturelles du Languedoc-Roussillon helene.palouzie@culture.gouv.fr 